\title{
Recursive modules for programming
}

\author{
Keiko Nakata \\ Kyoto University Research Institute for Mathematical Sciences \\ Jacques Garrigue \\ Graduate School of Mathematics, Nagoya University
}

\begin{abstract}
The ML module system is useful for building large-scale programs. The programmer can factor programs into nested and parameterized modules, and can control abstraction with signatures. Yet ML prohibits recursion between modules. As a result of this constraint, the programmer may have to consolidate conceptually separate components into a single module, intruding on modular programming. Introducing recursive modules is a natural way out of this predicament. Existing proposals, however, vary in expressiveness and verbosity. In this paper, we propose a type system for recursive modules, which can infer their signatures. Opaque signatures can also be given explicitly, to provide type abstraction either inside or outside the recursion. The type system is provably decidable, and is sound for a call-by-value semantics. We also gives a solution to the expression problem, in support of our design choices.
\end{abstract}




\section{Introduction}

When building a large software system, it is useful to decompose the system into smaller parts and to reuse them in different contexts. Module systems play an important role in facilitating such factoring of programs. Many modern programming languages provide some forms of module systems.

The family of ML programming languages, which includes SML[19] and Objective Caml [16], provides a powerful module system [17, 15]. Nested structures of modules allow hierarchical decomposition of programs. Functors can be used to express advanced forms of parameterization, which ease code reuse. Abstraction can be controlled by signatures with transparent, opaque or translucent types $[10,13]$.

In spite of this flexibility, the ML module language prohibits recursion between modules. This is a major disadvantage of ML, when compared to object-oriented languages, like Java. These languages have supported recursive definitions across class boundaries from the beginning, and this feature is heavily used in practice.

We, ML programmers, enjoy strong type safety. Yet, due to the lack of recursive modules, we may have to consolidate conceptually separate components into a single module, intruding on modular programming [24]. If we had both recursive modules and this flexible module language, we could enjoy a strongly type safe programming language with an equally strong expressive power.

Recently, much work has been devoted to investigating extensions with recursion of the ML module system. Two important issues involved are type checking and initialization. Crary, Harper and Puri [3], Russo [24], and Dreyer [5] have given type theoretic accounts for recursive modules. Boudol [1], Hirschowitz and Leroy [12], and Dreyer [4] have investigated type systems which guarantee well-formedness of recursive modules, ensuring that initialization of recursive modules will not attempt to access not-yet-evaluated values.

It seems that ML programmers are very close to use recursive modules in everyday programming. Indeed, some real ML family languages support recursive modules [16, 23], in which we can use them for practical programs, or, at least, get a flavor of them.

In this paper, we first review two examples. In the first one, two recursive modules Tree and Forest respect each other's privacy: we seal them with opaque signatures individually. Thus type abstraction is enforced inside the recursion. In the second, Tree and Forest are intimate: they know each other's exact implementations, and we seal them with an opaque signature as a whole. Thus type abstraction is enforced outside the recursion.

Both privacy and intimacy will be important for practical uses of recursive modules. Existing proposals, however, vary in their way to handle them. We may be 
denied privacy. We may have to write two different signatures for the same module; one of the signatures is solely for assisting the type checker and does not affect the resulting signature of the module.

Our goal is to develop a type system for recursive modules, which is practical and useful from the programmer's perspective; we want to use them easily in everyday programming, possibly combining with other constructs of the core and the module languages.

With this goal in mind, we propose a type system for recursive modules, in which modules can have privacy or intimacy depending on the situation they are in. The type system does not require additional signature annotations. Thus the programmer can either omit writing signatures or give signatures explicitly to control abstraction. Moreover, he can rely on type inference during development; all previous proposals by others do not support type inference for recursive modules.

In the paper, we also present an advanced example of recursive modules, by giving a concise and type safe solution to the expression problem [27]. In the example, we use recursive modules, applicative functors [14] and private row types [9] together. The example confirms that by combining recursive modules with other language constructions we can indeed enjoy a highly expressive power in a type safe and modular way.

Our contributions are summarized as follows.

- We examine two typical uses of recursive modules by giving concrete examples. These examples are useful for understanding basic uses of recursive modules.

- We propose a new type system for recursive modules with first-order applicative functors. The type system supports type inference for recursive modules, and is decidable and sound for a call-by-value semantics.

All examples we present in this paper are type checked in this type system, without requiring additional signature annotations.

- We give a type safe and concise solution to the expression problem, in order to demonstrate that recursive modules give us the highly expressive power in a modular way when combined with other language constructions.

The rest of the paper is organized as follows. In the next section, we review two examples of recursive modules and present the main features of our calculus, Traviata, used for our formal development. Section 3 gives the concrete syntax of Traviata. Section 4 and 5 explain the type system and present a soundness result. In Section 6, 
we give a solution to the expression problem. In Section 7, we examine the double vision problem [6]. Section 10 examines related work and Section 11 concludes. 


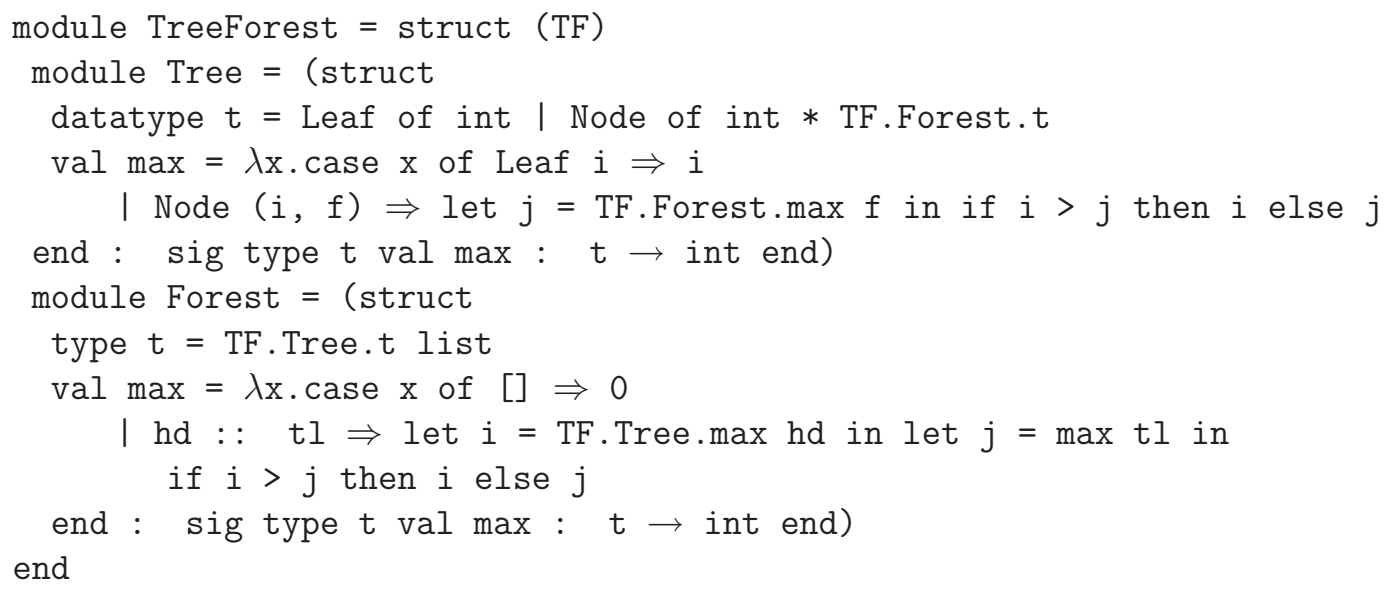

Figure 1: Modules for trees and forest

\section{Examples}

In this section, we review two examples to illustrate two possible uses of recursive modules and to informally present Traviata ${ }^{1}$.

The first example appears in Figure 1. The top-level module TreeForest contains two modules Tree and Forest: Tree represents a module for trees whose leaves and nodes are labeled with integers; Forest represents a module for unordered sets of those integer trees.

The modules Tree and Forest refer to each other in a mutually recursive way. Their type components Tree.t and Forest.t refer to each other, as do their value components Tree.max and Forest.max. These functions calculate the maximum integers a tree and a forest contain, respectively.

To enable forward references, we extend structures and signatures with implicitly typed declarations of self variables. Components of structures and signatures can refer to each other recursively using the self variables. For instance, TreeForest declares a self variable named TF, which is used inside Tree and Forest to refer to each other recursively. We keep the usual ML scoping rules for backward references. Thus Tree.max can refer to the Leaf and Node constructors without going through a self variable. Tree might also be used without prefix inside Forest, but the explicit notation seems clearer.

\footnotetext{
${ }^{1}$ In examples, we shall allow ourselves to use some usual core language constructions, such as let and if expressions and list constructors, even though they are not part of the formal development given in Section 3.
} 
This first example illustrates a possible use of recursive modules, where they respect each other's privacy. They are sealed with opaque signatures individually, enforcing type abstraction inside the recursion.

The second example appears in Figure 2. Now TreeForest is a functor, parameterized by the type of labels of trees. We assume that an applicative functor MakeSet is given in a library for making sets of comparable elements.

The modules Tree and Forest define the same recursive types as the first example, except that the argument types of the constructors Leaf and Node are parameterized. The module abbreviation module $\mathrm{F}=\mathrm{TF}$. Forest inside Tree allows us to use an abbreviation F for TF.Forest inside Tree. Similarly, the type $\mathbf{s}$ in Tree is an abbreviation which expands into TF.Forest.t.

In this second example, Tree and Forest are intimate: the functions Tree.split and Forest. sweep know the underlying implementations of the types Forest.t and Tree.t of the others, thus can construct and deconstruct values of those types. Given a tree, split cuts off the root node of the tree and returns the resulting forest. sweep gathers the leaves from a given forest.

Since the two modules are intimate, we do not seal Tree and Forest individually here. Instead, we seal them as a whole with an opaque signature. The signature only exposes functions split, sweep, and incr, which augments a given forest only if a given tree contains original labels that are not contained in the forest, but hides functions Tree.labels and Forest.labels, which are utility functions for incr. The signature also enforces type abstraction by hiding implementations of the types Tree.t and Forest.t, thus it protects privacy of the two modules from the outside.

The two examples we have seen so far illustrate two possible uses of recursive modules. They may have privacy, enforcing type abstraction inside the recursion. They may have intimacy, enforcing type abstraction outside the recursion. We think both uses are natural and would become common in practice.

Comparison with existing type systems The two examples presented are type checked in our type system without requiring additional annotations. Below, we examine the ways existing type systems handle these examples.

To avoid presenting too much annotations, we remove the module abbreviation module $F=T F$. Forest from Tree in Figure 2. Yet, although we can dispense with abbreviations by replacing them with their definitions altogether, they are useful in practice [21].

In Russo's system [24] there is no obvious way to type check the first example, keeping type abstraction between Tree and Forest. A suggested solution, which is 
found in his paper, is to annotate the self variable $\mathrm{TF}$ of TreeForest with a recursive signature $^{2} 3$ [24]:

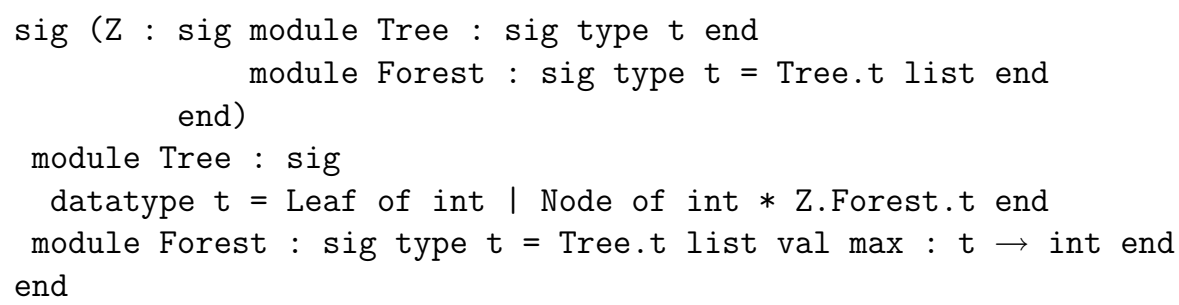

This annotation for TF, however, would break type abstraction between Tree and Forest, exposing underlying implementations of types Tree.t and Forest.t to each other.

In Dreyer's system [5], the sealing signatures for Tree and Forest must be given in advance. That is, the programmer has to write both signatures before defining either of the two modules, as opposed to Figure 1, where the signatures are written in a module-wise way.

O'Caml [16] type checks Figure 1 without modifications.

Next, we examine the second example. As we claimed, our type system type checks it without requiring additional annotations.

In Russo's system, the programmer must annotate TF with a recursive signature:

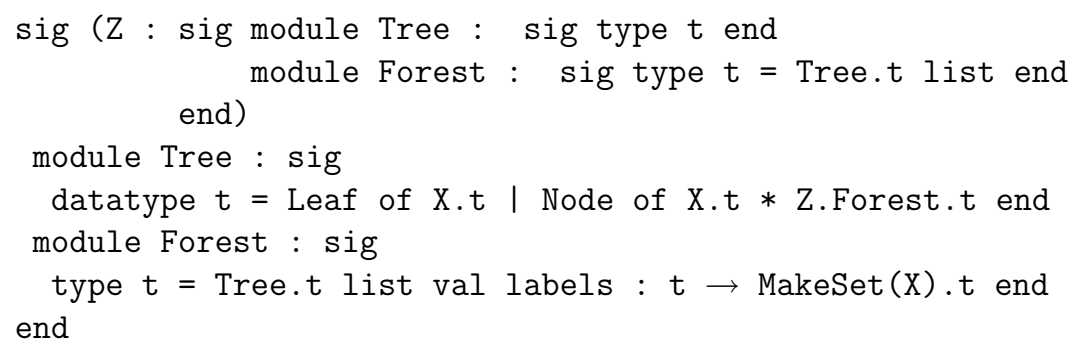

Note that this signature is solely for assisting the type checker. We have already given in Figure 2 the eventual signatures that Tree and Forest should have; these signatures do not reveal the underlying implementations of types Tree.t and Forest.t or the function Forest.labels.

To type check Figure 2 in Dreyer's system and O'Caml, the programmer must write fully manifesting signatures of Tree and Forest in advance, where the signatures declare every component of the modules. The type checker first type checks the

\footnotetext{
${ }^{2}$ This recursive signature does not exactly follow his syntax, e.g. we have to use the keyword structure instead of module in his system.

${ }^{3}$ We note that by permuting the definition order of Tree and Forest the amount of required annotations can be reduced to some extent in this case. However permutation does not always work.
} 
two modules assisted by these manifest signatures. Once this succeeds, type abstraction is enforced using the sealing signature given in Figure 2. Thus the programmer has to write annotations yet more verbose than in Russo's system.

We believe that both privacy and intimacy are important for practical uses of recursive modules. Existing type systems, however, do not handle them equally. These type systems may deny privacy. They may require additional annotations that are used only for helping the type checker, but do not affect resulting signatures of modules. Even if we assume that these annotations provide some useful information, our experience with type inference in ML is that one often writes a module without its signature, and then eventually writes a signature by editing the result of type inference. This technique has not been available with recursive modules in these type systems. 


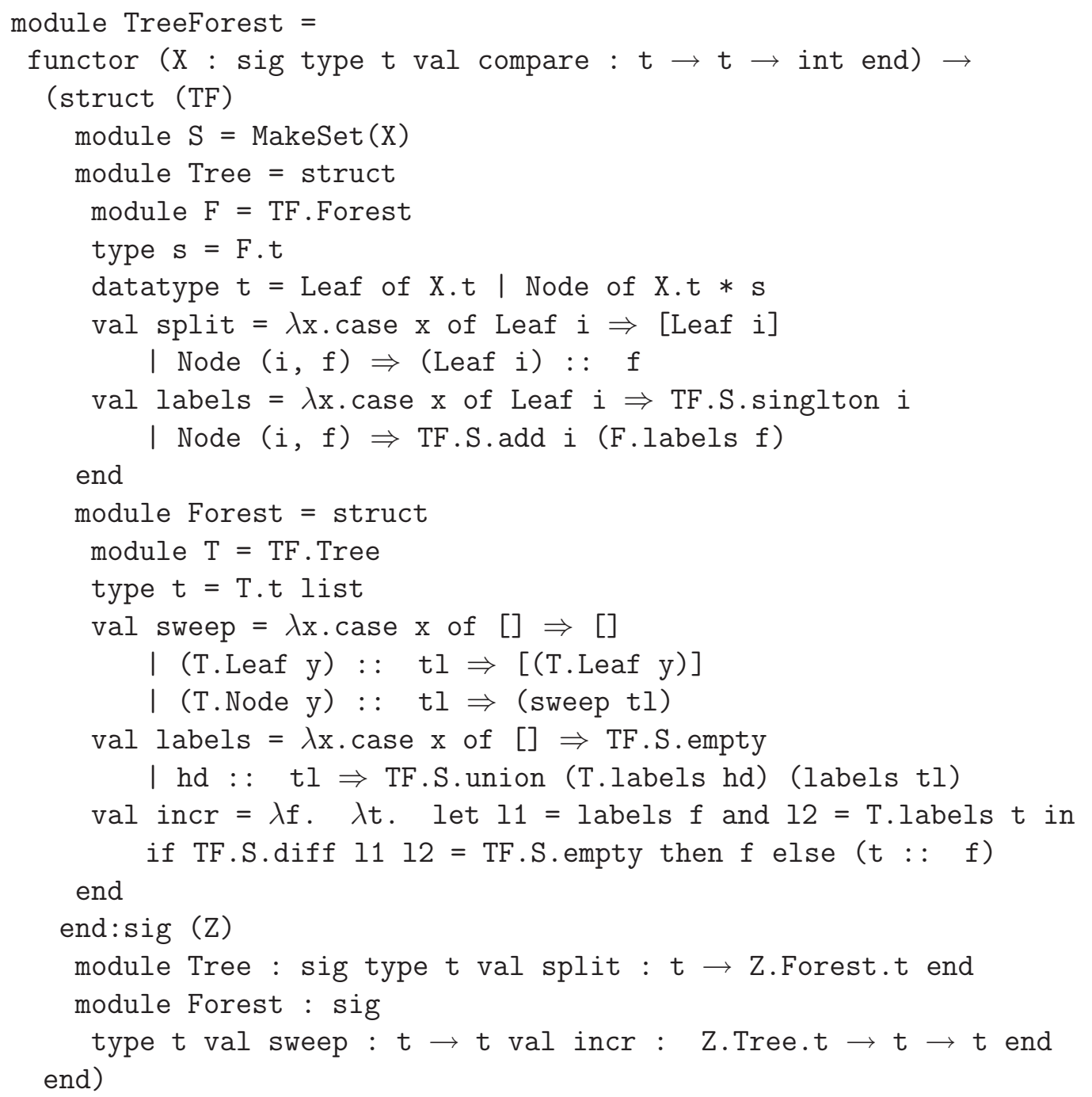

Figure 2: Intimate modules for trees and forests 


\section{Syntax}

Figure 3 gives the module language of Traviata, which is based on Leroy's applicative functor calculus [14]. We use $M$ as a metavariable for module names, $X$ for module variables and $Z$ for self variables. For simplicity, we distinguish them syntactically, however the context could tell them apart without this distinction. We also use $t$ for type names and $l$ for (core) value names.

For the purpose of both defining type equality and designing a decidable type system, we label module expressions, signatures and module variable signatures with integers. For instance, a module expression $E$ is a module expression description $E_{d}$ labeled with an integer $i$, where $E_{d}$ is either a structure, a functor, a sealing, a module identifier or a module variable ${ }^{4}$ One can think of the integer label $i$ of $E_{d}^{i}$ as the location of $E_{d}$ in the source program. For the interest of brevity, we may omit integer labels when they are not used. For the interest of clarity, we may write additional parentheses, for instance (functor $\left(\mathrm{X}:\right.$ sig type $\left.\left.t \mathrm{end}^{2}\right) \rightarrow \mathrm{X}^{3}\right)^{1}$. We use metavariables $i, j, k$ for integers.

As explained in the previous section, we extend structures and signatures with implicitly typed declarations of self variables to support recursive references. In the construct struct $(Z) D_{1} \ldots D_{n}$ end, the self variable $Z$ is bound in $D_{1} \ldots D_{n}$. Similarly, in the construct sig $(Z) B_{1} \ldots B_{n}$ end, the self variable $Z$ is bound in $B_{1} \ldots B_{n}$.

For simplicity, we provide different syntax for signatures and module variable signatures; the latter are used to specify signatures of functor arguments and do not declare self variables. In a practical system, we can unify their syntax for the programmer's benefit.

The construct which enables recursive references is recursive identifiers. A recursive identifier is constructed from a self variable and the dot notation ". $M$ ", which represents access to the sub-modules $M$ of a structure. A recursive identifier may begin from any bound self variable, and may refer to a module at any level of nesting within the recursive structure, regardless of component ordering. For instance, through the self variable of the top-level structure, one can refer to any module named in that structure except for those hidden within sealed sub-structures. It is important that recursive identifiers can only contain bound self variables, and that self variables of sealed modules are unbound outside them. Otherwise type abstraction could be broken.

\footnotetext{
${ }^{4}$ Note that Traviata does not have two different notions of opaque signatures and transparent ones.
} 
Module expression

$$
\text { E } \quad::=E_{d}^{i}
$$

Module expression descriptions

$$
\begin{aligned}
& E_{d} \quad::=\operatorname{struct}(Z) D_{1} \ldots D_{n} \text { end structure } \\
& \text { functor }(X: A) \rightarrow E \quad \text { functor } \\
& (E: S) \quad \text { sealing } \\
& \text { mid module identifier }
\end{aligned}
$$
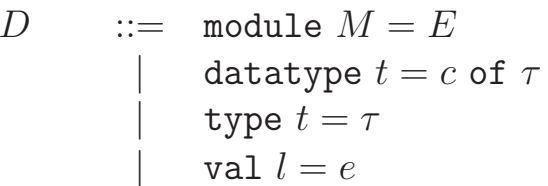

Signature

$S \quad::=S_{d}^{i}$

Signature descriptions

$S_{d} \quad::=\operatorname{sig}(Z) B_{1} \ldots B_{n}$ end

| functor $(X: A) \rightarrow S$

Module variable signature

$$
\text { A } \quad::=A_{d}^{i}
$$

Module variable signature description

$$
A_{d} \quad::=\operatorname{sig} B_{1} \ldots B_{n} \text { end }
$$

Specifications

$B \quad::=\operatorname{module} M: S$

$$
\left\{\begin{array}{l}
\text { datatype } t=c \text { of } \tau \\
\text { type } t=\tau \\
\text { type } t \\
\text { val } l: \tau
\end{array}\right.
$$

structure type functor type

Recursive identifiers

$$
\text { rid }::=Z \mid \text { rid.M }
$$

Module identifiers

$$
\text { mid } \quad:=\operatorname{rid}|\operatorname{mid}(\operatorname{mid})| \operatorname{mid}(X)
$$

Extended module identifiers

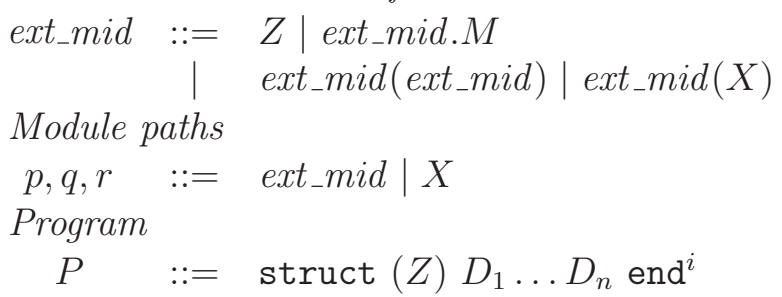

Figure 3: The module language of Traviata 


$$
\begin{array}{llll}
\text { Core types } & \tau & ::= & \mathbf{1}\left|\tau_{1} \rightarrow \tau_{2}\right| \tau_{1} * \tau_{2} \mid \text { p.t } \\
\text { Core expr. } & e & := & x|()|(\lambda x . e: \tau)\left|\left(e_{1}, e_{2}\right)\right| \pi_{i}(e) \mid e_{1}\left(e_{2}\right) \\
& & \mid & \text { rid.c } e|X . c e| \text { case } e \text { of } m s \mid \text { rid.l } \mid X . l \\
\text { Matching } & \text { ms } \quad::= & \text { rid.c } x \Rightarrow \text { e } \mid X . c x \Rightarrow e
\end{array}
$$

Figure 4: The core language of Traviata

For the sake of simplicity, functor applications only contain module identifiers and module variables.

To support applicative functors [14], we define a slightly extended class of identifiers, named module paths in Figure 3, which can liberally include functor applications. Core types defined in Figure 4 may use module paths. Applicative functors give us more flexibility in expressing type sharing constraint between recursive modules. In Section 6, we give a practical example which uses recursive modules and applicative functors together in support of our design choices. It will be useful to note that $Z \subseteq$ rid $\subseteq$ mid $\subseteq$ ext_mid $\subseteq$ p holds.

A program is a top-level structure which contains a bunch of recursive modules. In this paper, we only consider recursive modules, but not ordinary ones.

To obtain a decidable type system, we impose a first-order structure restriction that requires functors 1) not to take functors as argument, 2) or to access submodules of arguments. The first condition means that our functors are first-order, and the second implies that the programmer has to pass sub-modules as independent parameters for functors instead of passing a module which contains all of them. One might have noticed that the syntax of module expression descriptions excludes those of the forms $X . M$ and $X($ mid $)$. This is consistent with the restriction.

Figure 4 gives the our core language of Traviata. We use $x$ as a metavariable for program variables (variables, for short), and $c$ for value constructor names.

The core language describes a simple functional language extended with value paths X.l and rid.l, and type paths p.t. Value paths X.l and rid.l refer to the value components $l$ in the structures referred to by $X$ and rid, respectively. A type path p.t refers to the type component $t$ in the structure that $p$ refers to.

We may say paths to mean module, type and value paths as a whole.

An unusual convention is that a module variable is bound inside its own signature. For instance,

functor $(X:$ sig type $t$ val $1: X . t$ end $) \rightarrow X$

is a legal expression, which should be understood as

functor $(X:$ sig type $t$ val $l: t$ end $) \rightarrow X$ 
This convention is convenient when proving type soundness, as the syntax of paths is kept uniform, that is, every path is prefixed by either a self variable or a module variable. In Section 6, we give examples where this this convention is useful.

We write $M \operatorname{Vars}(p)$ to denote the set of module variables contained in the module path $p$. We also write $M \operatorname{Vars}(\tau), \operatorname{MVars}(e)$ and the likes with obvious meanings.

In the formalization, 1) function definitions are explicitly type annotated; 2) every structure and signature type but module variable signature declares a self variable; 3) a path is always prefixed by a self variable or a module variable. Our examples do not stick to these rules. Instead, we have assumed that there is an elaboration phase, prior to type checking, that adds type annotations for functions by running a type inference algorithm on the core language. The original program may still require some type annotations, to avoid running into the polymorphic recursion problem. In Section 9, we discuss the details of this inference algorithm. The elaboration phase also infers omitted self variables, to complete implicit backward references.

We assume that the following five conventions: 1) a program does not contain free module variables or free self variables; 2) all binding occurrences of module or self variables use distinct names; 3) any sequence of module definitions, type abbreviations, datatype definitions, value definitions, module specifications, manifest and opaque type specifications, datatype specifications and value specifications does not contain duplicate definitions or specifications for the same name; 4) all occurrences of module expressions, signatures and module variable signatures in a program are labeled with distinct integers; 5) module variable signatures do not contain module specifications. 


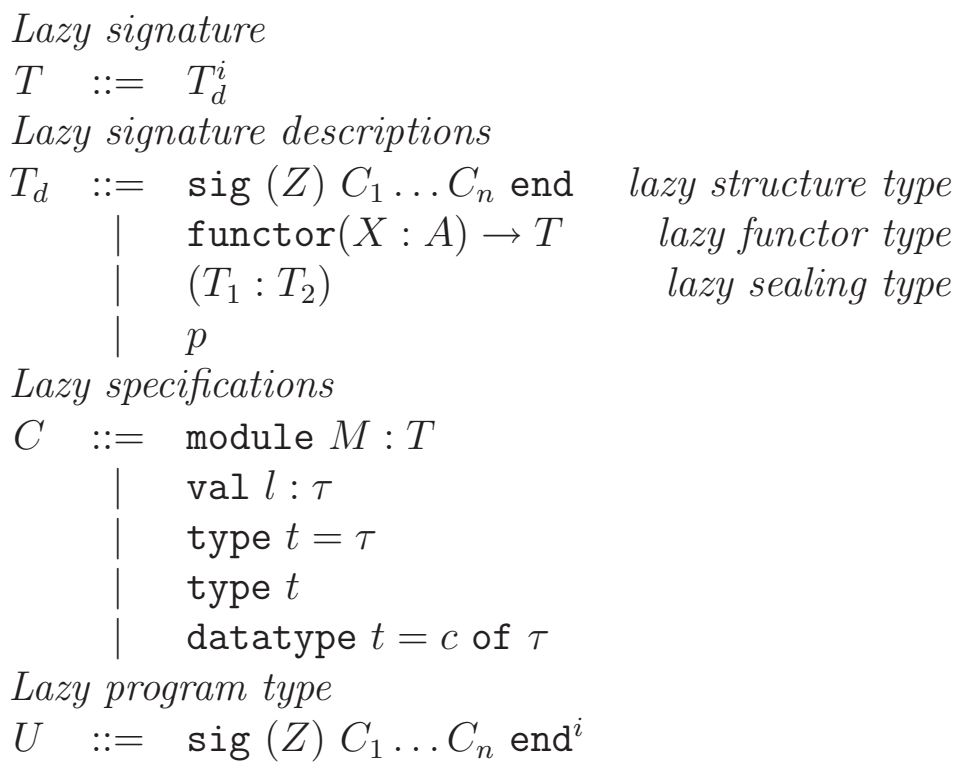

Figure 5: Lazy module types

\section{Reconstruction}

The type system is composed of two parts, namely a type reconstruction part and a type-correctness check part. Concretely, we type check a program $P$ in two steps: 1) reconstruct a lazy program type of $P$; at this point, we do not require the reconstructed type to be correct; 2 ) check type-correctness of $P$ by type checking $P$ in the intuitive way, using the result of the reconstruction in a type environment; once this second step is completed, we are certain both that $P$ is type-correct and that the reconstruction was correct.

In this section we describe the reconstruction part; the next section explains the type-correctness check part.

The rest of this section is organized as follows. In Section 4.1, we define lazy program types, which are output of the reconstruction algorithm. In Section 4.2, we define look-up judgment for using programs and lazy program types as lookup tables. In Section 4.3, we introduce "resolution algorithms", the key for enabling the reconstruction. Finally, in Section 4.5, we present an algorithm for reconstructing lazy program types from programs.

In the rest of the paper, we assume that self variables $Z$ are annotated with module variable environments $\theta$, written $Z^{\theta}$. A module variable environment is a substitution of module paths for module variables. Correspondingly, we assume that 


$$
\begin{aligned}
& \text { Top-levels } \quad O \quad::=P \mid U \\
& \text { Module descriptions } K, J::=E|S| A \mid T \\
& :=\quad::==\mid: \\
& \text { ss } \quad::=\text { struct } \mid \text { sig }
\end{aligned}
$$

Figure 6: Notation

each occurrence of a self variable in a program $P$ is implicitly annotated with an identity substitution $i d$. That is, we regard $Z$ as an abbreviation for $Z^{i d}$. We use $\theta$ as a metavariable for module variable environments.

\subsection{Lazy module types}

Figure 5 gives the syntax for lazy module types, which we use as signatures of modules during type checking. The syntax for lazy signature descriptions extends that for signature descriptions with the sealing construction $\left(T_{1}: T_{2}\right)$ and module paths. We use the sealing construction $\left(T_{1}: T_{2}\right)$ to check type-correctness of the sealing construction $(E: S)$ of module expression descriptions ((31) in Figure 18). We use module paths to instantiate signatures lazily $((61)$ in Figure 21$)$. In the construct $\operatorname{sig}(Z) C_{1} \ldots C_{n}$ end, the self variable $Z$ is bound in $C_{1} \ldots C_{n}$. A lazy program type is a top-level lazy structure type labeled with an integer. Note that lazy signatures include signatures.

We use the notation convention in Figure 6. In particular, we use $O$ as a metavariable for top-levels, which are either programs or lazy program types, and $K$ for module descriptions, which are either module expression descriptions, signature descriptions, module variable signature descriptions or lazy signature descriptions.

\subsection{Look-up}

Next, we define a look-up judgment for finding module descriptions and their integer labels from a top-level. During the reconstruction we use the judgment against programs; during the type-correctness check, we use the judgment against lazy program types.

We assume that, for a top-level $O$, there is a global mapping $\rho_{O}$ which sends i) a self variable $Z$ to the structure or the (lazy) structure type to which $Z$ is ascribed in $O$, and ii) a module variable $X$ to the module variable signature specified for $X$ in $O$. We say that in the construct struct $(Z) D_{1} \ldots D_{n}$ end $^{i}$ the self variable $Z$ is ascribed to struct $(Z) D_{1} \ldots D_{n}$ end $^{i}$. Similarly, in the constructs sig $(Z) B_{1} \ldots B_{n}$ end $^{i}$ 


$$
\begin{gathered}
\overline{O \vdash Z^{\theta} \mapsto\left(\theta, \rho_{O}(Z)\right)}(0) \quad \overline{O \vdash X \mapsto\left(i d, \rho_{O}(X)\right)}(1) \\
\frac{O \vdash p \mapsto\left(\theta, \text { ss } \ldots \text { module } M:=K^{j} \ldots \text { end }{ }^{i}\right) \quad K \neq\left(K_{1}^{k_{1}}: K_{2}^{k_{2}}\right)}{O \vdash p . M \mapsto\left(\theta, K^{j}\right)} \\
\frac{O \vdash p \mapsto\left(\theta, \text { ss } \ldots \text { module } M:=K^{j} \ldots \text { end }^{i}\right) \quad K=\left(K_{1}^{k_{1}}: K_{2}^{k_{2}}\right)}{O \vdash p . M \mapsto\left(\theta, K_{2}^{k_{2}}\right)} \\
\frac{O \vdash p_{1} \mapsto\left(\theta,\left(\text { functor }\left(X: A^{j}\right) \rightarrow K^{k}\right)^{i}\right) \quad K \neq\left(K_{1}^{k_{1}}: K_{2}^{k_{2}}\right)}{O \vdash p_{1}\left(p_{2}\right) \mapsto\left(\theta\left[X \mapsto p_{2}\right], K^{k}\right)}(4) \\
\frac{O \vdash p_{1} \mapsto\left(\theta,\left(\text { functor }\left(X: A^{j}\right) \rightarrow K^{k}\right)^{i}\right) \quad K=\left(K_{1}^{k_{1}}: K_{2}^{k_{2}}\right)}{O \vdash p_{1}\left(p_{2}\right) \mapsto\left(\theta\left[X \mapsto p_{2}\right], K_{2}^{k_{2}}\right)}
\end{gathered}
$$

Figure 7: Look-up

and sig $\left(Z^{\prime}\right) C_{1} \ldots C_{m}$ end $^{j}, Z$ and $Z^{\prime}$ are ascribed to sig $(Z) B_{1} \ldots B_{n}$ end $^{i}$ and $\operatorname{sig}\left(Z^{\prime}\right) C_{1} \ldots C_{m}$ end $^{j}$, respectively. The use of $\rho_{O}$ makes the presentation concise ${ }^{5}$.

We present inference rules for the look-up judgment in Figure 7 . The judgment $O \vdash p \mapsto\left(\theta, K^{i}\right)$ means that the module path $p$ refers to the module description $K$ labeled with the integer $i$ in the top-level $O$, where each module variable $X$ is bound to $\theta(X)$.

Let us examine each rule. For self variables and module variables, the judgment consults the global mapping $\rho_{O}$. Next two rules (3) and (4) handle module paths of the form $p . M$. A module path $p . M$ refers to the sub-module $M$ in the module that $p$ refers to. Hence $p$ must refer to either a structure or a (lazy) structure type. The rules (3) and (4) distinguish whether $M$ is bound to a sealing construction $\left(K_{1}^{j_{1}}: K_{2}^{j_{2}}\right)^{j}$ or not; when it is, then p.M resolves to the sealing part $K_{2}^{j_{2}}$. Thus, the judgment prevents peeking inside of sealed modules from the outside of them. The last two rules (5) and (6) handle module paths of the form $p_{1}\left(p_{2}\right)$. When $p_{1}$ refers to either a functor or a (lazy) functor type, then $p_{1}\left(p_{2}\right)$ resolves to the body of the functor, where the module variable environment is augmented with the new binding $\left[X \mapsto p_{2}\right]$. Again the rules (5) and (6) distinguish whether the body is a sealing construction or not.

\footnotetext{
${ }^{5}$ We could avoid this assumption of a global mapping by annotating each self variable with the source program location of the structure or structure type to which the self variable is ascribed. Since the source program can be regarded as a finite tree, we can represent every node of the tree by a finite representation (i.e., we need not use file names or line numbers.)
} 


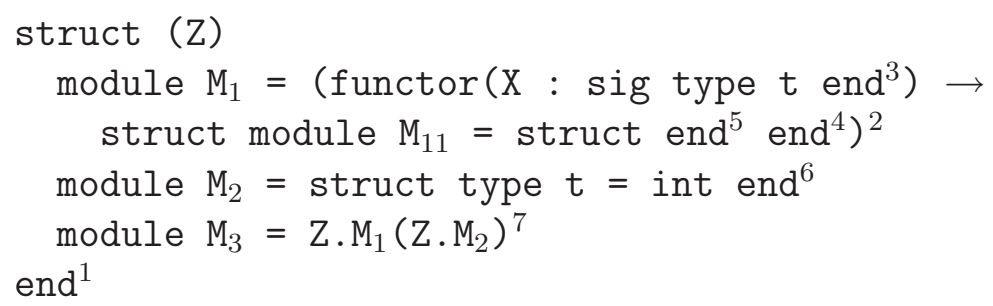

Figure 8: A program $\mathrm{P}_{1}$

The look-up judgment does not hold for arbitrary module paths. For instance, consider Figure 8. We have $\mathrm{P}_{1} \vdash \mathrm{Z} \cdot \mathrm{M}_{1}\left(\mathrm{Z} \cdot \mathrm{M}_{2}\right) \cdot \mathrm{M}_{11} \mapsto\left(\left[\mathrm{X} \mapsto \mathrm{Z} \cdot \mathrm{M}_{2}\right]\right.$, struct end $\left.{ }^{5}\right)$. But, the judgment does not hold for the module path Z.M.M.M. $M_{11}$.

Recall that we have assumed the absence of free module variables. This means that when $O \vdash p \mapsto\left(\theta, q^{i}\right)$, then $\operatorname{MVars}(q) \subseteq \operatorname{dom}(\theta)$. For a module variable environment $\theta, \operatorname{dom}(\theta)$ denotes the domain of $\theta$.

\subsection{Resolution algorithms}

Our type system differs from others in that it can resolve path references. Concretely, we developed a terminating procedure for determining the component that a path refers to, where the path may contain forward references. The motivation of this procedure was to define a decidable judgment for type equality. In a language with recursive modules and applicative functors, there is the potential that a program contains pathologically cyclic type abbreviations which may cause type equality check to diverge. We later noticed that a similar procedure enables type inference for recursive modules. Note that we cannot use the well-typedness of the source program when resolving path references, since we already need type equality to ensure this well-typedness.

We implement the procedure for path resolution as three algorithms, namely, a module path expansion algorithm PathExp, a type expansion algorithm TypExp and a core type reconstruction algorithm CtyInf. These algorithms use termination criteria based on ground term rewriting and recursive path ordering; the criteria do not rely on the well-typedness of the source program, and still allow flexible handling of module and type abbreviations.

In this paper, we do not explain these resolution algorithms in detail. There is another paper [20], which is devoted to their explanations. 
Located types We define a canonical form of types, called located types. The type system checks equality between two arbitrary types by reducing them into located types using TypExp.

A located type is a type composed of simple located types and $\mathbf{1}$ using $\rightarrow$ and $*$. Intuitively, a simple located type is an abstract type which is obtained by expanding all type and module abbreviations.

We first define located forms, a canonical form of module paths. A module path $p$ is in located form if and only if $p$ does not contain a module path which resolves to a module abbreviation.

Definition 1 A module path $p$ is in located form with respect to a top-level $O$ if and only if the following two conditions hold.

- $O \vdash p \mapsto\left(\theta, K^{i}\right)$ where $K$ is not a module path.

- For all $q$ in $\operatorname{args}(p), q$ is in located form.

For a module path $p, \operatorname{args}(p)$ denotes the set of module paths that $p$ contains as functor arguments, or:

$$
\begin{gathered}
\operatorname{args}\left(Z^{\theta}\right)=\bigcup_{X \in \operatorname{dom}(\theta)}\{\theta(X)\} \\
\operatorname{args}(p . M)=\operatorname{args}(p) \quad \operatorname{args}\left(p_{1}\left(p_{2}\right)\right)=\operatorname{args}\left(p_{1}\right) \cup\left\{p_{2}\right\}
\end{gathered}
$$

A simple located type is an abstract type whose prefix is a located form.

Definition 2 A simple located type with respect to a top-level $O$ is a type path p.t where $p$ is in located form with respect to $O$ and either $O \vdash p \mapsto(\theta$, ss . . datatype $t=$ $c$ of $\tau \ldots$ end $\left.{ }^{i}\right)$ or $O \vdash p \mapsto\left(\theta\right.$, ss ... type $t \ldots$ end $\left.^{i}\right)$ holds.

Now located types are defined below.

Definition 3 A located type with respect to a top-level $O$ is a type $\tau$ where each type $\tau^{\prime}$ in typaths $(\tau)$ is a simple located type with respect to $O$.

For a type $\tau$, typaths $(\tau)$ denotes the set of type paths that $\tau$ contains. Precisely,

$$
\operatorname{typaths}(\tau)=\left\{\begin{array}{cc}
\operatorname{typaths}\left(\tau_{1}\right) \cup \operatorname{typaths}\left(\tau_{2}\right) & \text { when } \tau=\tau_{1} \rightarrow \tau_{2} \\
& \text { or } \tau=\tau_{1} * \tau_{2} \\
\{p . t\} & \text { when } \tau=p . t \\
\emptyset & \text { when } \tau=\mathbf{1}
\end{array}\right.
$$




$$
\begin{aligned}
& \overline{O, \Sigma \vdash X \leadsto g} X \\
& \overline{O, \Sigma \vdash Z^{\theta} \leadsto g Z^{\theta}} \\
& O, \Sigma \vdash p \leadsto g p^{\prime} \\
& O, \Sigma \vdash p \leadsto g p^{\prime} \quad O \vdash p^{\prime} . M \mapsto\left(\theta, q^{i}\right) \\
& \frac{O \vdash p^{\prime} . M \mapsto\left(\theta, K^{i}\right) \quad K \notin \text { mid }}{O, \Sigma \vdash p \cdot M \sim_{g} p^{\prime} \cdot M} \quad \frac{q \neq X \quad O, \Sigma \uplus i \vdash q \sim{ }_{g} r}{O, \Sigma \vdash p \cdot M \sim_{g} \theta(r)} \\
& \frac{O, \Sigma \vdash p_{1} \sim_{g} p_{1}^{\prime} \quad O, \Sigma \vdash p_{2} \sim_{g} p_{2}^{\prime} \quad O \vdash p_{1}^{\prime}\left(p_{2}^{\prime}\right) \mapsto\left(\theta, K^{i}\right) \quad K \notin \text { mid }}{O, \Sigma \vdash p_{1}\left(p_{2}\right) \sim_{g} p_{1}^{\prime}\left(p_{2}^{\prime}\right)} \\
& O, \Sigma \vdash p_{1} \leadsto g p_{1}^{\prime} \quad O, \Sigma \vdash p_{2} \sim g p_{2}^{\prime} \\
& \frac{O \vdash p_{1}^{\prime}\left(p_{2}^{\prime}\right) \mapsto\left(\theta, q^{i}\right) \quad q \neq X \quad O, \Sigma \uplus i \vdash q \hookrightarrow_{g} r}{O, \Sigma \vdash p_{1}\left(p_{2}\right) \sim_{g} \theta(r)}
\end{aligned}
$$

Figure 9: Ground-normalization with respcet to $O$

$$
\begin{aligned}
& \eta_{O}\left(Z^{\theta}\right)=Z^{\theta^{\prime}} \\
& \text { where } \operatorname{dom}(\theta)=\operatorname{dom}\left(\theta^{\prime}\right) \text {, } \\
& \text { and, for all } X \in \operatorname{dom}(\theta), \theta^{\prime}(X)=\eta_{O}(\theta(X)) \\
& \eta_{O}(X)=\mathrm{X} \\
& \eta_{O}(p \cdot M)=\zeta_{O}\left(\eta_{O}(p) \cdot M\right) \\
& \eta_{O}\left(p_{1}\left(p_{2}\right)\right)=\zeta_{O}\left(\eta_{O}\left(p_{1}\right)\left(\eta_{O}\left(p_{2}\right)\right)\right) \\
& \zeta_{O}(p)= \begin{cases}\theta(X) & \text { when } O \vdash p \mapsto\left(\theta, X^{i}\right) \\
p & \text { otherwise }\end{cases}
\end{aligned}
$$

Figure 10: Variable normalization with respect to $O$ 


\subsubsection{Module path expansion}

We define the module path expansion algorithm PathExp by composing ground normalization and variable normalization, which are defined below.

We define the ground normalization in Figure 9. The judgment $O, \Sigma \vdash p \sim_{g} q$ means that the ground normalization expands $p$ into $q$ where $\Sigma$ is locked, with respect to the top-level $O$. We use $\Sigma$ as a metavariable for sets of integers. The notation $\Sigma \uplus i$ means $\Sigma \cup\{i\}$ whenever $i \notin \Sigma$. Note that derivations of the ground normalization are deterministic. In particular, it is an error state when there are no applicable rules.

We define the variable normalization with respect to a top-level $O$ using functions $\eta_{O}$ and $\zeta_{O}$, found in Figure 10.

Then we define PathExp such that it takes as argument a top-level $O$ and a module path $p$, then either returns a module path $q$ when $O, \emptyset \vdash p \leadsto{ }_{g} p^{\prime}$ and $\eta_{O}\left(p^{\prime}\right)=q$ hold or else raises an error when this cannot be done.

Definition 4 A module path $p$ (resp. a type $\tau$ and an expression e) has located variables if and only if all the self variables contained in $p$ (resp. $\tau$ and $e$ ) are in located form.

Since $Z^{\text {id }}$ is in located form, all module paths, types and expressions appearing in the source program have located variables.

Proposition 1 ([20]) For any top-level $O$ and module path $p$ having located variables, if $\operatorname{Path} \operatorname{Exp}(O, p)=q$, then $q$ is in located form with respect to $O$.

\subsubsection{Type expansion}

We define the type expansion algorithm in Figure 11. The judgment $O, \Omega \vdash \tau_{1} \downarrow \tau_{2}$ means that the algorithm expands the type $\tau_{1}$ into the type $\tau_{2}$ where $\Omega$ is locked,with respect to the top-level $O$. We use $\Omega$ as a metavariable for pairs $(i, t)$ of an integer $i$ and a type name $t$.

Then we define TypExp such that it takes as argument a top-level $O$ and a type $\tau$, then either returns a type $\tau^{\prime}$ when $O, \emptyset \vdash \tau \downarrow \tau^{\prime}$ holds or else raises an error when no rule applies, i.e. it cannot prove that the input type does not contain cyclic or dangling references.

Proposition 2 ([20]) For any top-level $O$ and type $\tau$ having located variables, Typ $\operatorname{Exp}(O, \tau)$ either returns a located type with respect to $O$ or else raises an error. 


$$
\begin{aligned}
& \overline{O, \Omega \vdash \mathbf{1 \downarrow 1}} \\
& \frac{O, \Omega \vdash \tau_{1} \downarrow \tau_{1}^{\prime} \quad O, \Omega \vdash \tau_{2} \downarrow \tau_{2}^{\prime}}{O, \Omega \vdash \tau_{1} \rightarrow \tau_{2} \downarrow \tau_{1}^{\prime} \rightarrow \tau_{2}^{\prime}} \quad \frac{O, \Omega \vdash \tau_{1} \downarrow \tau_{1}^{\prime} \quad O, \Omega \vdash \tau_{2} \downarrow \tau_{2}^{\prime}}{O, \Omega \vdash \tau_{1} * \tau_{2} \downarrow \tau_{1}^{\prime} * \tau_{2}^{\prime}} \\
& \frac{\operatorname{PathExp}(O, p)=p^{\prime} \quad O \vdash p^{\prime} \mapsto\left(\theta, \text { ss . . type } t \ldots \text { end }^{i}\right)}{O, \Omega \vdash p . t \downarrow p^{\prime} . t} \\
& \frac{\operatorname{PathExp}(O, p)=p^{\prime} \quad O \vdash p^{\prime} \mapsto\left(\theta, \text { ss } \ldots \text { datatype } t=c \text { of } \tau \ldots \text { end }^{i}\right)}{O, \Omega \vdash p . t \downarrow p^{\prime} . t} \\
& P a t h \operatorname{Exp}(O, p)=p^{\prime} \quad O \vdash p^{\prime} \mapsto\left(\theta, \text { ss . . type } t=\tau_{1} \ldots \text { end }^{i}\right) \\
& \frac{O, \Omega \uplus(i, t) \vdash \tau_{1} \downarrow \tau_{2} \quad O, \Omega \vdash \theta\left(\tau_{2}\right) \downarrow \tau}{O, \Omega \vdash p . t \downarrow \tau}
\end{aligned}
$$

Figure 11: Type expansion with respect to $O$

$$
\begin{aligned}
& \overline{P, \Psi, \Gamma \vdash x \triangleright \Gamma(x)} \quad \overline{P, \Psi, \Gamma \vdash() \triangleright \mathbf{1}} \quad \frac{P, \Psi, \Gamma \vdash e_{1} \triangleright \tau_{1} \quad P, \Psi, \Gamma \vdash e_{2} \triangleright \tau_{2}}{P, \Psi, \Gamma \vdash\left(e_{1}, e_{2}\right) \triangleright \tau_{1} * \tau_{2}} \\
& \frac{P, \Psi, \Gamma \vdash e \triangleright \tau_{1} * \tau_{2}}{P, \Psi, \Gamma \vdash \pi_{i}(e) \triangleright \tau_{i}} \quad \frac{P, \Psi, \Gamma \vdash e_{1} \triangleright \tau^{\prime} \rightarrow \tau}{P, \Psi, \Gamma \vdash e_{1}\left(e_{2}\right) \triangleright \tau} \quad \frac{\operatorname{Typ} \operatorname{Exp}\left(P, \tau^{\prime}\right)=\tau}{P, \Psi, \Gamma \vdash\left(\lambda x . e: \tau^{\prime}\right) \triangleright \tau} \\
& \frac{\operatorname{PathExp}(P, p)=p^{\prime} \quad \gamma\left(O, p^{\prime}, c\right)=\left(t, \tau_{1}\right)}{P, \Psi, \Gamma \vdash p . c \text { e } \triangleright p^{\prime} . t} \\
& \frac{\operatorname{PathExp}(P, p)=p^{\prime} \quad \gamma\left(O, p^{\prime}, c\right)=\left(t, \tau_{1}\right) \quad P, \Psi, \Gamma, x: \tau_{1} \vdash e_{2} \triangleright \tau}{P, \Psi, \Gamma \vdash \text { case } e_{1} \text { of } p . c x \Rightarrow e_{2} \triangleright \tau} \\
& \operatorname{PathExp}(P, p)=p^{\prime} \quad P \vdash p^{\prime} \mapsto\left(\theta, \operatorname{struct} \ldots \operatorname{val} l=e \ldots \text { end }^{i}\right) \\
& P, \Psi \uplus(i, l), \emptyset \vdash e \triangleright \tau_{1} \quad \operatorname{Typ} \operatorname{Exp}\left(P, \theta\left(\tau_{1}\right)\right)=\tau \\
& P, \Psi, \Gamma \vdash p . l \triangleright \tau \\
& \operatorname{PathExp}(P, p)=p^{\prime} \\
& \frac{P \vdash p^{\prime} \mapsto\left(\theta, \operatorname{sig} \ldots \operatorname{val} l: \tau^{\prime} \ldots \text { end }{ }^{i}\right) \quad \operatorname{Typ} \operatorname{Exp}\left(P, \theta\left(\tau^{\prime}\right)\right)=\tau}{P, \Psi, \Gamma \vdash p . l \triangleright \tau}
\end{aligned}
$$

Figure 12: Core type reconstruction with respect to $P$ 


$$
\begin{aligned}
& \gamma(O, p, c)=(t, \tau) \text { when } \\
& O \vdash p \mapsto\left(\theta, \text { ss } \ldots \text { datatype } t=c \text { of } \tau^{\prime} \ldots \text { end }{ }^{i}\right) \text { and } \operatorname{Typ} \operatorname{Exp}\left(O, \theta\left(\tau^{\prime}\right)\right)=\tau
\end{aligned}
$$

Figure 13: Datatype look-up with respect to $O$

\subsection{Core type reconstruction}

We define the core type reconstruction algorithm in Figure 12. The judgment $P, \Psi, \Gamma \vdash e \triangleright \tau$ means that the algorithm reconstructs the type $\tau$ for the expression $e$ where $\Psi$ is locked, with respect to the program $P$. We use $\Psi$ as a metavariable for pairs $(i, l)$ of an integer $i$ and a value name $l$.

Then we define CtyInf such that it takes as argument a program $P$ and a core expression $e$, then either returns a type $\tau$ when $P, \emptyset, \emptyset \vdash e \triangleright \tau$ holds or else raises an error when this cannot be done.

Proposition 3 ([20]) For any program $P$ and core expression e having located variables, CtyInf $(P, e)$ either returns a located type with respect to $P$ or else raises an error.

For a program $P$ and a core expression $e, \operatorname{Cty} \operatorname{Inf}(P, e)$ returns a located type that $e$ would have when $e$ is type-correct, but it does not check that $e$ is indeed type-correct. For instance, for an expression $e_{1}\left(e_{2}\right)$, CtyInf only reconstructs a type of $e_{1}$, which must be of the form $\tau_{1} \rightarrow \tau_{2}$, then returns the result type $\tau_{2}$; it does not check that $e_{2}$ has a type which is equivalent to $\tau_{1}$. In Section 5 , we explain the type-correctness check part of the type system. Type-correctness of $e_{1}\left(e_{2}\right)$ is ensured in that part.

\subsection{Lazy program type reconstruction algorithm}

Figure 14 presents inference rules for the lazy program type reconstruction algorithm with respect to a program $P$. The algorithm uses CtyInf (see (10)), hence it does not ensure type-correctness of the program $P$. It either returns a lazy program type that $P$ would have when $P$ is type-correct, or else raises an error when it cannot prove that $P$ does not contain cyclic or dangling references. Note that it does not check type-correctness of functor applications (see (16)).

Then we define Reconst $P$ such that it takes a program $P$ as argument, then either returns $U$ when $P \vdash P \triangleright U$ holds or else raises an error when this cannot be done. 
Definitions and Specifications

$$
\begin{aligned}
& \frac{P \vdash E \triangleright T}{P \vdash \text { module } M=E \triangleright \text { module } M: T} \\
& \frac{P \vdash S \triangleright T}{P \vdash \text { module } M: S \triangleright \text { module } M: T} \\
& \frac{\operatorname{Typ} \operatorname{Exp}(P, \tau)=\tau^{\prime}}{P \vdash \text { datatype } t=c \text { of } \tau \triangleright \text { datatype } t=c \text { of } \tau^{\prime}} \\
& \frac{\operatorname{Cty} \operatorname{Inf}(P, e)=\tau}{P \vdash \operatorname{val} l=e \triangleright \operatorname{val} l: \tau}(10) \quad \frac{\operatorname{Typ} \operatorname{Exp}(P, \tau)=\tau^{\prime}}{P \vdash \operatorname{val} l: \tau \triangleright \operatorname{val} l: \tau^{\prime}} \\
& \frac{P \vdash E_{d} \triangleright T_{d}}{P \vdash E_{d}^{i} \triangleright T_{d}^{i}} \\
& \frac{P \vdash D_{1} \triangleright C_{1} \ldots P \vdash D_{n} \triangleright C_{n}}{P \vdash \operatorname{struct}(Z) D_{1} \ldots D_{n} \text { end } \triangleright \operatorname{sig}(Z) C_{1} \ldots C_{n} \text { end }} \\
& \frac{P \vdash A \triangleright A^{\prime} \quad P \vdash E \triangleright T}{P \vdash \text { functor }(X: A) \rightarrow E \triangleright \text { functor }\left(X: A^{\prime}\right) \rightarrow T} \\
& \frac{P \vdash E \triangleright T_{1} \quad P \vdash S \triangleright T_{2}}{P \vdash(E: S) \triangleright\left(T_{1}: T_{2}\right)} \\
& \overline{P \vdash p \triangleright p} \\
& \frac{P \vdash S_{d} \triangleright T_{d}}{P \vdash S_{d}^{i} \triangleright T_{d}^{i}}
\end{aligned}
$$$$
\text { (8) } \frac{\operatorname{Typ} \operatorname{Exp}(P, \tau)=\tau^{\prime}}{P \vdash \text { type } t=\tau \triangleright \text { type } t=\tau^{\prime}}
$$

Signature descriptions

$$
\begin{gathered}
\frac{P \vdash B_{1} \triangleright C_{1} \ldots P \vdash B_{n} \triangleright C_{n}}{P \vdash \operatorname{sig}(Z) B_{1} \ldots B_{n} \text { end } \triangleright \operatorname{sig}(Z) C_{1} \ldots C_{n} \text { end }} \\
P \vdash A \triangleright A^{\prime} P \vdash S \triangleright T \\
\frac{P \vdash \text { functor }(X: A) \rightarrow S \triangleright \text { functor }\left(X: A^{\prime}\right) \rightarrow T}{P}
\end{gathered}
$$

Module variable signature

$$
\frac{P \vdash A_{d 1} \triangleright A_{d 2}}{P \vdash A_{d 1}^{i_{1}} \triangleright A_{d 2}^{i_{2}}}
$$

Module variable signature description

$$
\frac{P \vdash B_{1} \triangleright B_{1}^{\prime} \ldots P \vdash B_{n} \triangleright B_{n}^{\prime}}{P \vdash \operatorname{sig} B_{1} \ldots B_{n} \text { end } \triangleright \operatorname{sig} B_{1}^{\prime} \ldots B_{n}^{\prime} \text { end }}
$$

Figure 14: Lazy signature reconstruction with respect to $P$ 
Proposition 4 For any program $P$, Reconst $P(P)$ either returns a lazy program type or raises an error.

Usually, we write $U_{P}$ to denote the lazy program type such that $\operatorname{Reconst} P(P)=$ $U_{P}$ holds. 


$$
\frac{U \vdash \operatorname{Typ} \operatorname{Exp}\left(U, \tau_{1}\right) \equiv_{\tau} \operatorname{Typ} \operatorname{Exp}\left(U, \tau_{2}\right)}{U \vdash \tau_{1} \equiv \tau_{2}}
$$

Figure 15: Type equivalence with respect to $U$

\section{Type system}

One of the main difficulties in type checking recursive modules is how to reason about forward references. Usually, a type checker consults a type environment for the necessary type information about paths. When paths only contain backward references, it is sufficient to accumulate in the type environment signatures of previously type checked modules. When modules are defined recursively, however, paths may contain forward references. Then the type checker may attempt to ask the type environment for a signature of a module which is not yet type checked.

To circumvent difficulties arising from forward references, other type systems rely on signature annotations. As we examined in Section 2, this requirement compels the programmer to write two different signatures for the same module to enforce type abstraction outside the recursion. Moreover, the programmer cannot rely on type inference during development due to it. This is unfortunate since a lot of useful inference algorithms have been and will be developed to support smooth development of programs.

We have a reconstruction algorithm, hence we do not need the assistance of signature annotations. That is, we use the result of reconstruction as type environment instead of using programmer-supplied annotations.

There are three tasks to be completed in this type-correctness check part: 1) to check type-correctness of core expressions. (Recall that CtyInf does not ensure typecorrectness of expressions that it reconstructs types for.); 2) to check well-formedness of module paths, that is, to check that functor applications contained in the paths are type-correct and that references of the paths are not cyclic or dangling; 3) to check that, for every sealing construction $(E: S)$, the module expression $E$ inhabits the signature $S$.

\subsection{Type equality}

We define a type equivalence judgment in Figure 15, with auxiliaries in Figure 16 and 17. The judgment $\tau_{1} \vdash \tau_{2} \equiv$ means that two types $\tau_{1}$ and $\tau_{2}$ are equivalent with respect to the lazy program type $U$. We check equivalence between two arbitrary types by reducing them into located types. 


$$
\begin{gathered}
\frac{U \vdash \tau_{1} \equiv_{\tau} \tau_{1}^{\prime}}{U \vdash \mathbf{1} \equiv_{\tau} \mathbf{1}}(23) \quad U \vdash \tau_{2} \equiv_{\tau} \tau_{2}^{\prime} \\
\frac{U \vdash \tau_{1} \equiv_{\tau} \tau_{1}^{\prime}}{U \vdash \tau_{2} \equiv_{\tau} \tau_{1}^{\prime} \rightarrow \tau_{2}^{\prime}}(24) \tau_{2} \equiv_{\tau} \tau_{2}^{\prime} \\
U \vdash \tau_{1} * \tau_{2} \equiv_{\tau} \tau_{1}^{\prime} * \tau_{2}^{\prime}
\end{gathered}
$$

Figure 16: Equivalence on located types with respect to $U$

$$
\begin{gathered}
U \vdash p_{1} \mapsto\left(\theta_{1}, T_{d 1}^{i_{1}}\right) \quad U \vdash p_{2} \mapsto\left(\theta_{2}, T_{d 2}^{i_{2}}\right) \quad i_{1}=i_{2} \\
\forall X \in \operatorname{dom}\left(\theta_{1}\right), U \vdash \theta_{1}(X) \equiv_{p} \theta_{2}(X) \\
U \vdash p_{1} \equiv_{p} p_{2}
\end{gathered}
$$

Figure 17: Equivalence on located forms with respect to $U$

Figure 16 defines equivalence on located types. The first three rules are straightforward. The last rule judges whether two abstract types are equivalent. Two types $p_{1} . t$ and $p_{2} . t$ are equivalent if and only their prefixes $p_{1}$ and $p_{2}$ are equivalent module paths. (Note that since $p_{1} . t$ is a located type, $p_{1}$ is in located form.)

Figure 17 defines a judgment for equivalence on module paths in located form. Two located forms $p_{1}$ and $p_{2}$ are equivalent if and only if they refer to module descriptions at the same location (i.e., labeled with the same integer) and their functor arguments are equivalent. Take a look at the look-up judgment (Figure 7) again. The module variable environment $\theta_{1}$ collects all module paths contained in $p_{1}$ as functor arguments.

\subsection{Typing rules}

In Figure 18 and 19, we present typing rules for the type-correctness check, with auxiliaries in Figure 20, 21 and 22.

The judgment $U \vdash E: T$ means that the module expression $E$ of lazy signature $T$ is type-correct with respect to the lazy program type $U$. The judgment $e, \Gamma \vdash \tau$ : means that the core expression $e$ of type $\tau$ is type-correct under the type environment $\Gamma$ with respect to $U$. A type environment assigns a located type to a variable. Other judgments are read similarly.

The typing rules in Figure 18 are mostly straightforward. Here we only explain the rule for sealing.

The rule (31) checks that the sealing construction $(E: S)$ is type-correct. In particular, the third premise is for ensuring that the module expression $E$ inhabits the signature $S$; it checks that the lazy signature $T_{1}$ of $E_{1}$ is a subtype of $\operatorname{Subst}\left(T_{1}, S\right)$. 
Module expression

$$
\frac{U \vdash E_{d}: T_{d}}{U \vdash E_{d}^{i}: T_{d}^{i}}(28)
$$

Module expression descriptions

$$
\begin{gathered}
U \vdash D_{1}: C_{1} \ldots U \vdash D_{n}: C_{n} \\
\frac{U \vdash \operatorname{struct}(Z) D_{1} \ldots D_{n} \text { end }: \operatorname{sig}(Z) C_{1} \ldots C_{n} \text { end }}{U: A^{\prime} U \vdash E: T} \\
\frac{U \vdash \operatorname{functor}\left(X: A^{\prime}\right) \rightarrow T}{U \vdash \text { functor }(X: A) \rightarrow E \text { f }}(30) \\
\frac{U \vdash E: T_{1} \quad U \vdash S: T_{2} \quad T_{1} \vdash S u b s t\left(T_{1}, S\right)<}{U \vdash(E: S):\left(T_{1}: T_{2}\right)}(31) \quad \frac{U \vdash p \text { wf }}{U \vdash p: p}(32)
\end{gathered}
$$

Definitions and Specifications

$$
\begin{aligned}
& \begin{array}{c}
\frac{U \vdash E: T}{U \vdash \text { module } M=E: \text { module } M: T}(33) \quad \frac{e, \emptyset \vdash}{U \vdash \operatorname{val} l=e} \\
\frac{U \vdash \tau \diamond \operatorname{Typ} \operatorname{Exp}(U, \tau)=\tau^{\prime}}{U \vdash \text { datatype } t=c \text { of } \tau: \text { datatype } t=c \text { of } \tau^{\prime}}
\end{array} \\
& \frac{U \vdash \tau \diamond \operatorname{Typ} \operatorname{Exp}(U, \tau)=\tau^{\prime}}{U \vdash \text { type } t=\tau: \text { type } t=\tau^{\prime}} \\
& \overline{U \vdash \text { type } t \text { : type } t} \\
& \frac{U \vdash S: T}{U \vdash \text { module } M: S: \text { module } M: T} \\
& \frac{U \vdash \tau \diamond \operatorname{Typ} \operatorname{Exp}(U, \tau)=\tau^{\prime}}{U \vdash \operatorname{val} l: \tau: \operatorname{val} l: \tau^{\prime}}
\end{aligned}
$$

Signature

$$
\frac{U \vdash S_{d}: T_{d}}{U \vdash S_{d}^{i}: T_{d}^{i}}(40)
$$

Signature descriptions

$$
\begin{gathered}
\frac{U \vdash B_{1}: C_{1} \ldots U \vdash B_{n}: C_{n}}{U \vdash \operatorname{sig}(Z) B_{1} \ldots B_{n} \text { end }: \operatorname{sig}(Z) C_{1} \ldots C_{n} \text { end }} \\
\frac{U \vdash A: A^{\prime} U \vdash S: T}{U \vdash \text { functor }(X: A) \rightarrow S: \text { functor }\left(X: A^{\prime}\right) \rightarrow T}
\end{gathered}
$$

Module variable signature

$$
\frac{U \vdash A_{d 1}: A_{d 2}}{U \vdash A_{d 1}^{i}: A_{d 2}^{i}}(43)
$$

Module variable signature description

$$
\frac{U \vdash B_{1}: B_{1}^{\prime} \ldots U \vdash B_{n}: B_{n}^{\prime}}{U \vdash \operatorname{sig} B_{1} \ldots B_{n} \text { end }: \operatorname{sig} B_{1}^{\prime} \ldots B_{n}^{\prime} \text { end }}
$$

Figure 18: Typing rules for the module language with respect to $U$ 


$$
\begin{aligned}
& \text { Core types } \\
& \overline{U \vdash \mathbf{1} \diamond}(45) \quad \frac{U \vdash \tau_{1} \diamond U \vdash \tau_{2} \diamond}{U \vdash \tau_{1} \rightarrow \tau_{2} \diamond}(46) \quad \frac{U \vdash \tau_{1} \diamond U \vdash \tau_{2} \diamond}{U \vdash \tau_{1} * \tau_{2} \diamond}(47) \\
& \frac{U \vdash p \text { wf } \quad T y p \operatorname{Exp}(U, p . t)=\tau}{U \vdash p . t \diamond}(48) \\
& \text { Core expressions } \\
& \overline{U, \Gamma \vdash(): \mathbf{1}}(49) \quad \frac{x \in \operatorname{dom}(\Gamma)}{U, \Gamma \vdash x: \Gamma(x)}(50) \\
& \frac{U, \Gamma \vdash e_{1}: \tau_{1} \quad U, \Gamma \vdash e_{2}: \tau_{2}}{U, \Gamma \vdash\left(e_{1}, e_{2}\right): \tau_{1} * \tau_{2}}(51) \quad \frac{U, \Gamma \vdash e: \tau_{1} * \tau_{2}}{U, \Gamma \vdash \pi_{i}(e): \tau_{1}} \\
& \frac{U \vdash \tau \diamond \quad \operatorname{Typ} \operatorname{Exp}(U, \tau)=\tau_{1} \rightarrow \tau_{2} \quad U, \Gamma, x: \tau_{1} \vdash e: \tau_{3} \quad U \vdash \tau_{2} \equiv \tau_{3}}{U, \Gamma \vdash(\lambda x . e: \tau): \tau_{1} \rightarrow \tau_{2}} \\
& \frac{U, \Gamma \vdash e_{1}: \tau_{1} \rightarrow \tau_{2} \quad U, \Gamma \vdash e_{2}: \tau_{3} \quad U \vdash \tau_{1} \equiv \tau_{3}}{U, \Gamma \vdash e_{1}\left(e_{2}\right): \tau_{2}} \\
& \frac{U \vdash p \mathrm{wf} \quad \operatorname{PathExp}(U, p)=p^{\prime} \quad \gamma\left(U, p^{\prime}, c\right)=\left(t, \tau_{1}\right) \quad U, \Gamma \vdash e: \tau_{2} \quad U \vdash \tau_{1} \equiv \tau_{2}}{U, \Gamma \vdash p . c e: p^{\prime} . t} \\
& U, \Gamma \vdash e_{1}: \tau_{1} \quad U \vdash p \text { wf } \operatorname{PathExp}(U, p)=p^{\prime} \\
& \frac{\gamma\left(U_{P}, p^{\prime}, c\right)=\left(t, \tau_{2}\right) \quad U \vdash \tau_{1} \equiv p^{\prime} . t \quad U, \Gamma, x: \tau_{2} \vdash e_{2}: \tau}{U, \Gamma \vdash \text { case } e_{1} \text { of } p . c x \Rightarrow e_{2}: \tau}(56) \\
& U \vdash p \text { wf } \operatorname{PathExp}(U, p)=p^{\prime} \\
& \frac{U \vdash p^{\prime} \mapsto\left(\theta, \operatorname{sig} \ldots \operatorname{val} l: \tau^{\prime} \ldots \text { end }^{i}\right) \quad \operatorname{Typ} \operatorname{Exp}\left(U, \theta\left(\tau^{\prime}\right)\right)=\tau}{U, \Gamma \vdash p . l: \tau}
\end{aligned}
$$

Figure 19: Typing rules for the core language with respect to $U$ 
$\operatorname{Subst}\left(T_{d}^{i}, S_{d}^{j}\right)=\operatorname{subst1}\left(T_{d}, S_{d}\right)^{j}$

$\operatorname{subst1}\left(p, \operatorname{sig}(Z) B_{1} \ldots B_{n}\right.$ end $)$

$=\left(\operatorname{sig}(Z) \operatorname{subst2}\left(p,[Z \mapsto p] B_{1}\right) \ldots \operatorname{subst2}\left(p,[Z \mapsto p] B_{n}\right)\right.$ end $)$

$\operatorname{subst1}\left(p, \operatorname{functor}(X: A) \rightarrow S_{d}^{i}\right)$

$=\operatorname{functor}(X: A) \rightarrow \operatorname{subst1}\left(p(X), S_{d}\right)^{i}$

$\operatorname{subst1}\left(\operatorname{sig}(Z) C_{1} \ldots C_{n}\right.$ end, sig $\left(Z^{\prime}\right) B_{1} \ldots B_{m}$ end)

$=\operatorname{sig}\left(Z^{\prime}\right)$

$\operatorname{subst3}\left(C_{\sigma(1)},\left[Z^{\prime} \mapsto Z\right] B_{1}\right) \ldots$ subst3 $\left(C_{\sigma(m)},\left[Z^{\prime} \mapsto Z\right] B_{m}\right)$ end

$\operatorname{subst1}(\operatorname{sig} \ldots$ end, functor $(X: A) \rightarrow S)=$ raise Error

$\operatorname{subst1}\left(\right.$ functor $(X: A) \rightarrow T_{d}^{i}$, functor $\left.\left(X^{\prime}: A^{\prime}\right) \rightarrow S_{d}^{j}\right)$

$=$ functor $\left(X^{\prime}: A^{\prime}\right) \rightarrow \operatorname{subst1}\left(\left[X \mapsto X^{\prime}\right] T_{d}, S_{d}\right)^{j}$

$\operatorname{subst1}($ functor $(X: A) \rightarrow T$, sig...end) $=$ raise Error

$\operatorname{subst1}\left(\left(T: T_{d}^{i}\right), S_{d}\right)=\operatorname{subst1}\left(T_{d}, S_{d}\right)$

$\operatorname{subst2}\left(p\right.$, module $\left.M: S_{d}^{i}\right)=\operatorname{module} M: \operatorname{subst1}\left(p \cdot M, S_{d}\right)^{i}$

$\operatorname{subst2}(p, B)=B \quad$ where $B$ is not a module specification

subst3(module $M: T_{d}^{i}$, module $\left.M: S_{d}^{j}\right)=$ module $M: \operatorname{subst1}\left(T_{d}, S_{d}\right)^{j}$

$\operatorname{subst3}(C, B)=B \quad$ where $B$ is not a module specification

Figure 20: Substitution 


$$
\begin{aligned}
& \frac{U \vdash T_{d}<S_{d}}{U \vdash T_{d}^{i}<S_{d}^{j}}(58) \quad \frac{U \vdash T_{d}<A_{d}}{U \vdash T_{d}^{i}<A_{d}^{j}} \\
& \frac{U \vdash T_{d}<S_{d}}{U \vdash\left(T: T_{d}^{i}\right)<S_{d}}(60) \\
& \frac{\operatorname{PathExp}(U, p)=p^{\prime} \quad U \vdash p^{\prime} \mapsto\left(\theta, T_{d}^{i}\right) \quad U \vdash \theta\left(T_{d}\right)<S_{d}}{U \vdash p<S_{d}} \\
& \frac{\sigma:\{1, \ldots, m\} \mapsto\{1, \ldots, n\} \quad \forall i \in\{1, \ldots, m\}, U \vdash C_{\sigma(i)}<B_{i}}{U \vdash \operatorname{sig}(Z) C_{1} \ldots C_{n} \text { end }<\operatorname{sig}\left(Z^{\prime}\right) B_{1} \ldots B_{m} \text { end }} \\
& \frac{U \vdash A^{\prime}<A \quad U \vdash\left[X \mapsto X^{\prime}\right] T<S}{U \vdash \text { functor }(X: A) \rightarrow T<\text { functor }\left(X^{\prime}: A^{\prime}\right) \rightarrow S} \\
& \frac{\sigma:\{1, \ldots, m\} \mapsto\{1, \ldots, n\} \quad \forall i \in\{1, \ldots, m\}, U \vdash C_{\sigma(i)}<B_{i}}{U \vdash \operatorname{sig} C_{1} \ldots C_{n} \text { end }<\operatorname{sig} B_{1} \ldots B_{m} \text { end }} \\
& \overline{U \vdash \text { type } t<\text { type } t} \\
& \text { (65) } \\
& \overline{U \vdash \text { type } t=\tau<\text { type } t} \\
& \begin{array}{c}
U \vdash \tau_{1} \equiv \tau_{2} \\
\frac{U \vdash \text { datatype } t=c \text { of } \tau_{1}<\text { datatype } t=c}{U \vdash T<S} \\
\frac{U \vdash \text { module } M: T<\text { module } M: S}{U-S}
\end{array}
\end{aligned}
$$

Figure 21: Subtyping with respect to $U$

$$
\begin{aligned}
& \overline{U \vdash X \mathrm{wf}}(72) \quad \frac{U \vdash p \mathrm{wf} \quad \operatorname{PathExp}(U, p . M)=q}{U \vdash p \cdot M \mathrm{wf}} \\
& U \vdash p_{1} \text { wf } U \vdash p_{2} \text { wf } \\
& \operatorname{PathExp}\left(U, p_{1}\right)=p_{1}^{\prime} \quad \operatorname{PathExp}\left(U, p_{2}\right)=p_{2}^{\prime} \quad \operatorname{PathExp}\left(U, p_{1}\left(p_{2}\right)\right)=q \\
& \frac{\left.U \vdash p_{1}^{\prime} \mapsto\left(\theta, \text { functor }\left(X: A_{d}^{j}\right) \rightarrow T\right)^{i}\right) \quad p_{2}^{\prime} \vdash \theta\left[X \mapsto p_{2}^{\prime}\right]\left(A_{d}\right)<}{U \vdash p_{1}\left(p_{2}\right) \text { wf }}
\end{aligned}
$$

Figure 22: Well-formed module paths with respect to $U$ 
The subtyping relation, to be given below, follows that of Leroy's applicative functor calculus. In particular, for two manifest type specifications type $t: \tau_{1}$ and type $t: \tau_{2}$ to be in subtyping relations, $\tau_{1}$ and $\tau_{2}$ must be equivalent. To check type equivalence, the type system expand types using TypExp; here is the reason that we define the function Subst, which is found in Figure 20.

The function Subst performs explicit substitution for self variables declared inside sealing signatures. For instance, consider Figure 2. The reconstruction algorithm infers that the function split in Forest has a type TF. Tree.t $\rightarrow$ TF. Tree.t list (For clarity, we add omitted self variables.). The sealing signature specifies that split has a type Z.Tree.t $\rightarrow$ Z.Forest.t. Both the reconstructed type and the specified type are located types, but they are not equivalent according to the type equivalence judgment. In fact, for Forest to inhabit the sealing signature, the reconstructed type TF.Tree.t $\rightarrow$ TF. Tree.t list should be equivalent to TF. Tree.t $\rightarrow \mathrm{TF}$.Forest.t, which is the type obtained by substituting TF for $\mathrm{Z}$ in the specified type $Z$.Tree.t $\rightarrow Z$.Forest.t. Indeed, this is satisfied since the type TF.Forest.t expands into TF.Tree.t list.

One may think that it would be more natural to identify signatures related by $\alpha$ renaming rather than to perform explicit substitution. Yet implicit renaming makes it complex to use the type expansion algorithm, which is developed separately from the typing rules.

In Figure 21, we define subtyping relations between a lazy signature and a signature (58), between a lazy signature and a module variable signature (59), between a lazy signature description and a signature description ((60) to (63)), between a lazy signature description and a module variable description $((64))$ and between a lazy specification and a specification $((65)$ to $(71))$. The rules are mostly intuitive. The reader should only look at the rule (61). A lazy signature description can be a module path $p$. To check that $p$ is a subtype of a signature description $S_{d}$, we instantiate the lazy signature of the module that $p$ refers to; we use the module path expansion algorithm PathExp to resolve $p$ 's reference. For the decidability of the subtyping relations, it is important that only lazy signature descriptions can be module paths, but ordinary signature descriptions cannot.

The judgment $U \vdash p$ wf, defined in Figure 22, checks that the module path $p$ is well-formed. For instance, the rule (32) in Figure 18 uses this judgment for checking type-correctness of module paths. The judgment ensures that the module path $p$ does not contain cyclic or dangling references and that functor applications contained in $p$ are type-correct.

Definition 5 A program $P$ is well-typed if and only if $\operatorname{Reconst} P(P)=U$ and $U \vdash$ 
$P: U$ holds.

Proposition 5 For any program $P$, it is decidable whether $P$ is well-typed or not. 


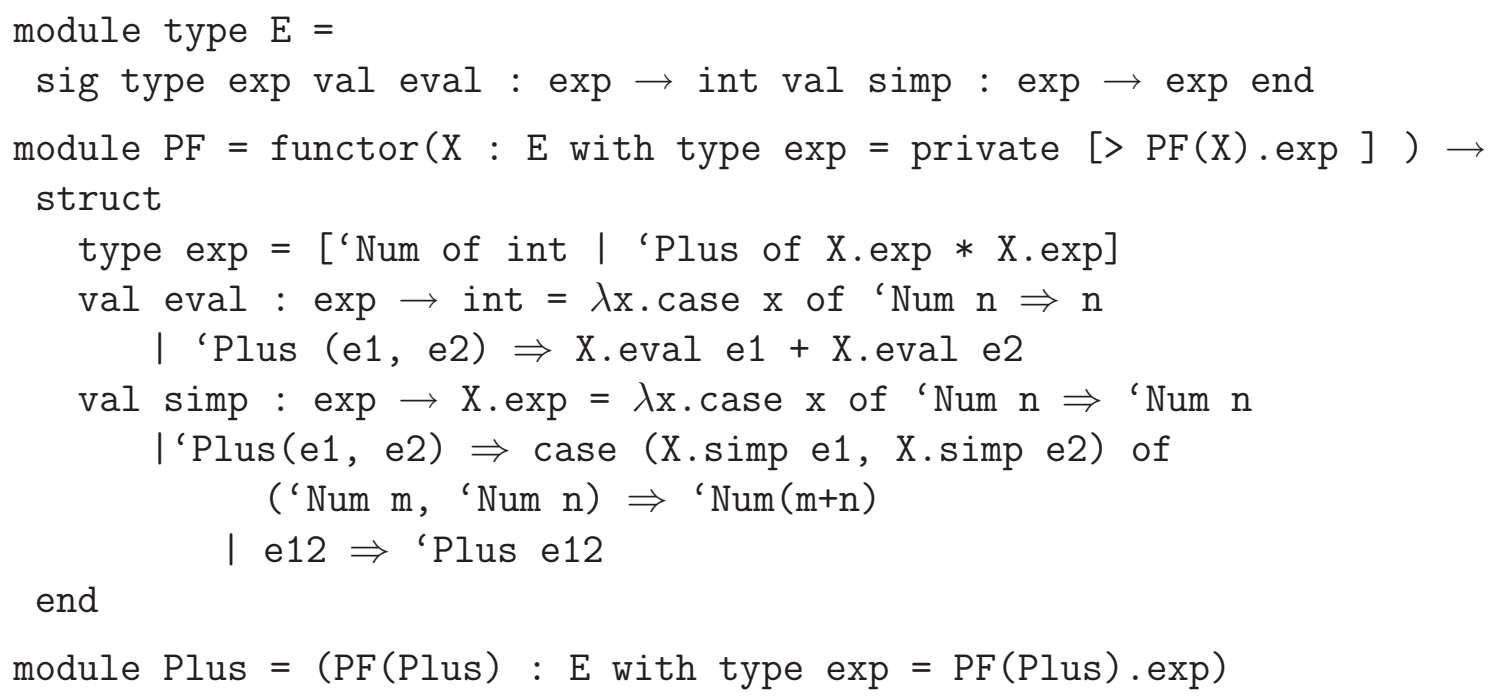

Figure 23: A first language

\section{The expression problem}

In this section, we present an advanced example of recursive modules, by giving a solution to the expression problem [27].

The expression problem, originally coined by Phil Wadler, dates back to Cook, who first discussed this problem [2]. It is one of the most fundamental problems one faces during the development of extensible software. Here, we paraphrase a typical example of this problem in the following way: suppose that we have a small expression language, composed of a recursively defined datatype and processors which operate on this datatype; then we want to extend the expression language in two dimensions, that is, to extend the datatype with new constructors and to add new processors that operate on both existing and new constructors. That a programming language can solve this problem in a type safe and concise way has been regarded as a measure of the expressive power of the language. Many researchers have addressed themselves to this problem, using different programming languages $[22,28,26]$.

Our aim here is not to draw a conclusion that our solution is better than other solutions. It is not easy to compare the quality of different solutions, without deep understandings of each implementation language that is used to express each solution. Instead, we aim to give a useful example of recursive modules, in order to show that by combining recursive modules with other constructs of the core and the module languages we can obtain more expressive power in a modular way. 
The example we use here extends the one in [9]. It is a variation on the expression problem, where we only insist on the addition of new constructors. Adding new processors is easy in this setting.

We shall assume that we have extended Traviata with polymorphic variants [7], private row types [9] and some usual module language constructions. Adding polymorphic variants and private row types is straightforward. We add typing rules for them to our language. Allowing structures to contain module type definitions may not be easy, but having module type definitions in the top-level is easy.

To reduce notational burden, we omit, here and elsewhere, preceding self variables even for forward references when no ambiguity seems to arise. We also omit the toplevel struct and end.

We define our first expression language in Figure 23, using the functor PF. The type exp defined in the body of PF indicates that the first language supports expressions composed of integer constants and addition. The function eval is for evaluating the expressions into integers. The function simp is for simplifying the expressions, by reducing the 'Plus constructor into the 'Num constructor when possible.

To keep the first language extensible, we leave PF open recursion; the polymorphic variant type exp and functions eval and simp recur through PF's parameter X.

The intuition of the example is that PF takes as argument an expression language which is built by extending the addition language that PF defines. This is exactly what the signature of $X$ expresses; here is the key of the example. The type specification type $t=$ private $[>P F(X)$.exp] specifies an abstract type into which the type $P F(X)$. exp can be coerced, or, informally, an abstract type which is a supertype of $P F(X)$.exp. The type $P F(X)$.exp refers to the type exp defined inside PF's body. Hence X's signature specifies that PF can only be applied to a module whose defining expression language supports both integers and addition. This recursive use of $\mathrm{PF}(\mathrm{X})$. exp to constrain PF's argument is the main difference with the solution in [9]. By avoiding the need to define types outside of the functor, it allows for a more concise and scalable solution. Observe that if it were not for all of applicative functors, private row types and flexible path references, we could not write X's signature in this way.

The use of polymorphic variants, which are structural types unlike usual nominal datatypes, is important also for defining the function simp. The function simp has the type $\exp \rightarrow \mathrm{X}$. exp. Since the type $\mathrm{X}$. exp structurally contains the type exp, as specified in the X's signature, all of 'Num $n$, 'Num $(m+n)$ and 'Plus e12, which are the results of the case branches, are citizens of the type $\mathrm{X}$. exp.

The module Plus instantiates the addition language, by closing PF's open re- 


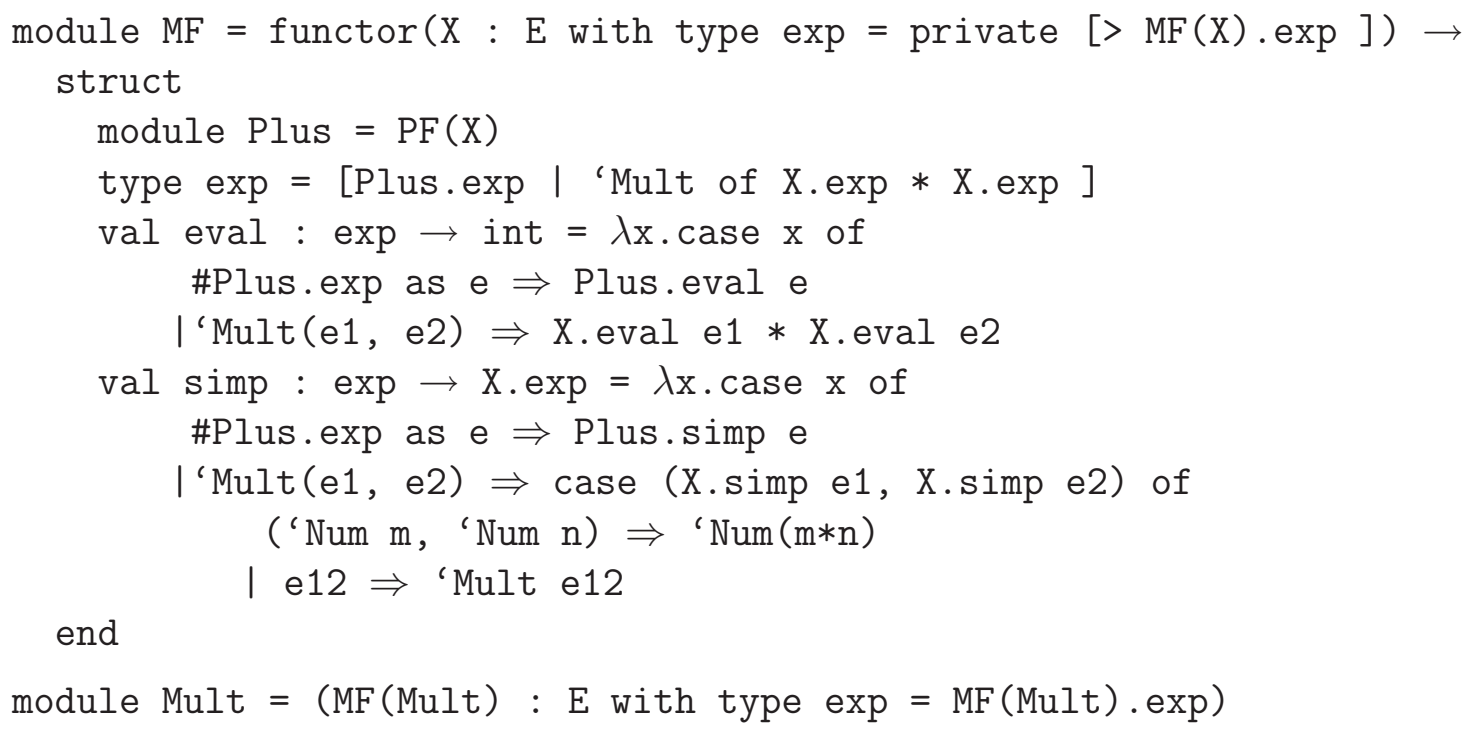

Figure 24: A second language

cursion. Observe that both the type and the value level open recursion are closed simultaneously, that is, by taking the fix-point of PF, the forwardings X.exp, X. eval and $\mathrm{X}$. simp are connected to exp, simp and eval themselves, thus yielding self contained recursive type exp and recursive functions eval and simp.

Now we can perform addition on the first language. For instance,

val e1 = Plus.eval ('Plus ('Num 3, 'Num 4))

Next, we define our second expression language using the functor MF in Figure 24. The second language supports expressions composed of multiplication and addition on integer constants.

We use the exactly same idiom as the first language to define this second language. In particular, the type $M F(X)$. exp appearing in X's signature refers to the type exp defined in the body of MF.

Note that we instantiate the first addition language inside MF, and use it in functions eval and simp to delegate known cases by variant dispatch. Thus we avoid duplication of program codes.

The module Mult instantiates the second language, by closing MF's open recursion. Now we can do arithmetic on the second language. For instance,

val e2 = Mult.eval ('Plus('Mult('Num 3, 'Num 4), 'Num 5))

Finally, we demonstrate in Figure 25 that it is easy to compose independent 


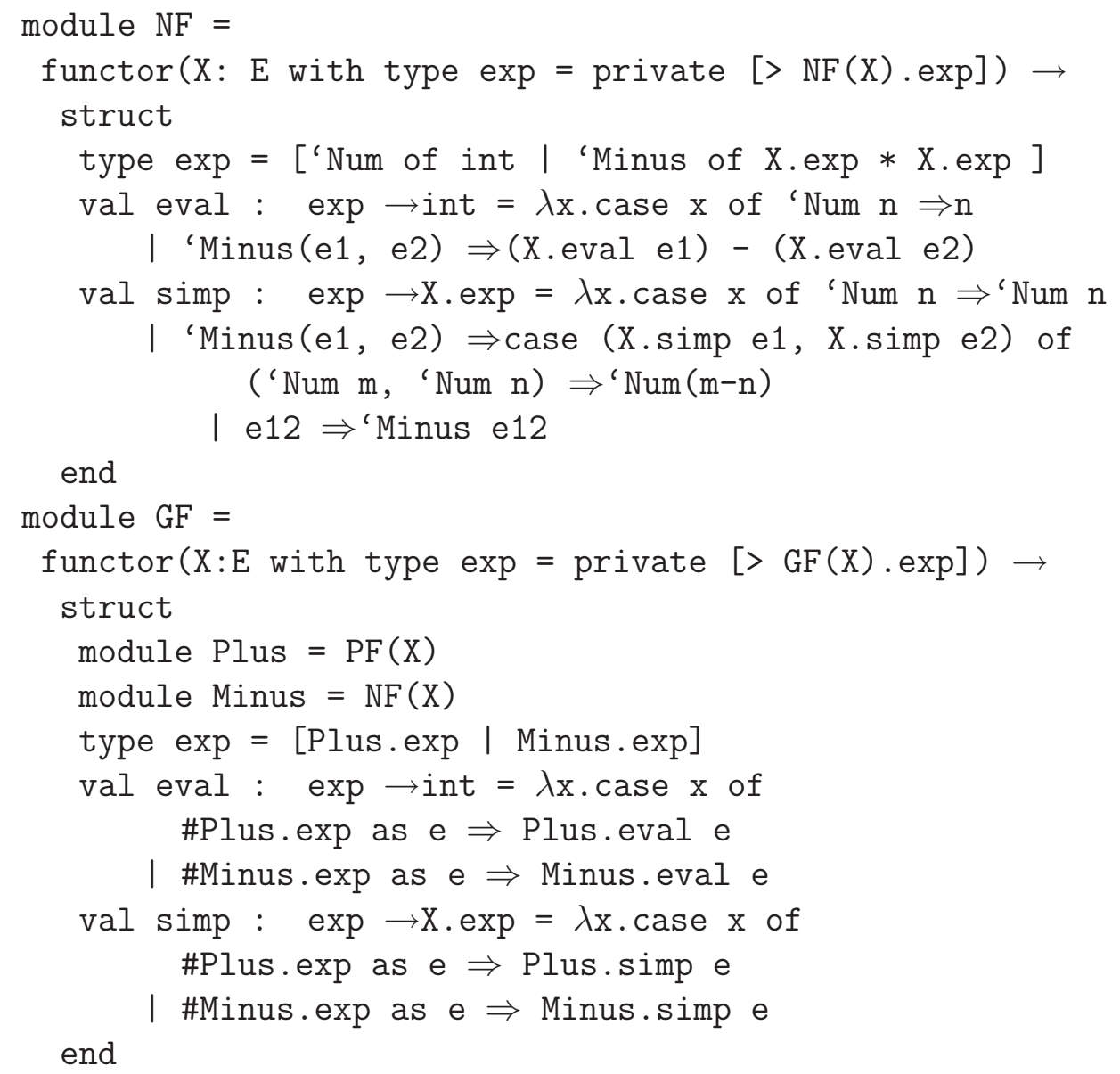

Figure 25: To merge independantly developed extensions 
extensions into a single expression language.

Having seen examples here and in Section 2, we confirm that recursive modules are useful in several situations. Moreover, when combined with other language constructions, they give us the highly expressive power in a modular way. We believe that recursive modules are a promising candidate for supporting robust extensible software. 


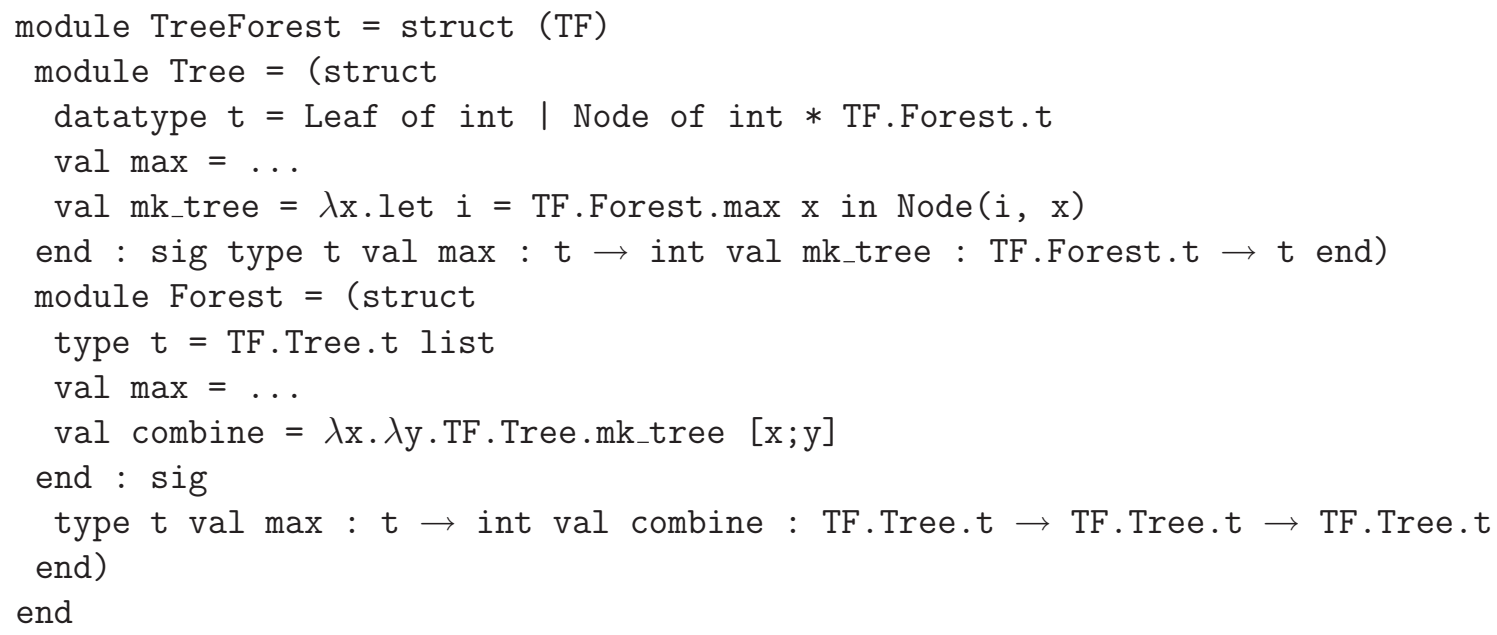

Figure 26: An expample for the double vision problem

\section{The double vision problem}

Here we examine the double vision problem [6], a typing difficulty involved in recursive modules, in the context of Traviata. Detailed examinations of this problem are found in $[6,3]$.

\subsection{The situation we have to handle}

When a module is sealed with a signature, the type system distinguishes the module defined inside the signature and the module which inhabits the signature. For instance, consider Figure 1. Inside Forest, the type $t$ and the type TF.Forest.t are not equivalent; the former is an internal type, which refers to Forest's type $t$ inside the sealing, but the latter is an external type, which refers to Forest's type $t$ outside.

This design choice of type equivalence keeps the type equivalence judgment simple. Yet, it might be occasionally inconvenient, for instance, when the programmer wants to build a value of an external type inside sealing.

To see a concrete situation, consider Figure 26. This is the same program as in Figure 1, but here Tree and Forest contain new functions mk_tree and combine, respectively; the former is for building a tree from a given forest and the latter for building a tree from given two trees.

Our type system cannot type check the defining expression of combine. For the 
expression $[\mathrm{x} ; \mathrm{y}]$ inside the body of combine, the core type reconstruction algorithm infers that the expression has a type TF. Tree.t list; the function TF. Tree.mk_tree takes an argument of type TF.Forest.t, which is specified in Tree's sealing signature. According to our type equivalence judgment, however, the types TF.Forest.t and $\mathrm{TF}$. Tree.t list are not equivalent, since TF.Forest.t is an abstract type thus is not equivalent to any other types than itself.

This kind of situation typically occurs when the programmer attempts to cyclically import, inside a sealed module, a value that is exported by the same module as a value of an abstract type. Note that such reimportation is only possible with recursive modules, but not with ordinary modules.

\subsection{Type coercion}

Currently we provide a core language construction, called type coercion, that allows the programmer to coerce types of expressions from internal types to external types and vice versa, in an explicit way. The type coercion construction is of the form $\left(e: \tau::>\tau^{\prime}\right.$ ), which informally reads as "to coerce the type $\tau$ of the expression $e$ into $\tau^{\prime \prime \prime}$. For instance, the programmer can define a type-correct combine as

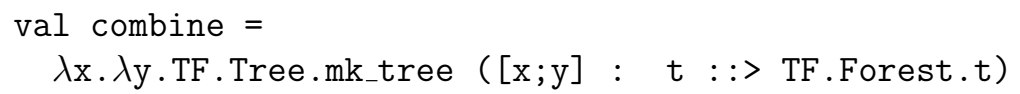

(Observe that the internal type $t$ of Forest is only visible inside Forest.)

In the rest of this section, we formalize type coercion.

Note that the programmer can avoid this situation by providing the following module Ty inside Forest, instead of providing the type definition type $t=$ TF. Tree.t list as in Figure 26.

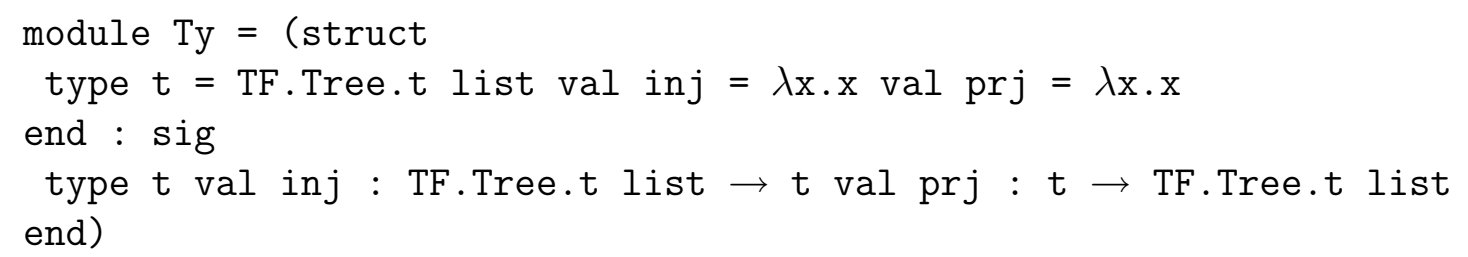

The programmer needs to use the functions inj and $p r j$ as needed when defining functions Forest.max and Forest.combine. By hiding inj and prj from Tree, but only exporting the abstract type Ty. $t$, he can produce the same effect as in Figure 26 without type errors.

Locating paths First, we introduce locating paths. Intuitively, a locating path is an absolute module path which refers to a module relative to the top-level, whereas 


$$
\begin{aligned}
& \overline{O \vdash \epsilon \mapsto_{l}(i d, O)} \\
& \frac{O \vdash l c \mapsto_{l}\left(\theta, \text { ss } \ldots \text { module } M:=K^{j^{\prime}} \ldots \text { end }^{j}\right) \quad K \neq\left(K_{1}^{k_{1}}: K_{2}^{k_{2}}\right)}{O \vdash[\text { lc. } M, 0] \mapsto_{l}\left(\theta, K^{j^{\prime}}\right)} \\
& \frac{O \vdash l c \mapsto\left(\theta,\left(\text { functor }\left(X: A^{j_{2}}\right) \rightarrow K^{j_{3}}\right)^{j_{1}}\right) \quad K \neq\left(K_{1}^{k_{1}}: K_{2}^{k_{2}}\right)}{O \vdash[l c(p), 0] \mapsto_{l}\left(\theta[X \mapsto p], K^{j_{3}}\right)} \\
& \frac{O \vdash l c \mapsto_{l}\left(\theta, \text { ss } \ldots \text { module } M:=K^{j^{\prime}} \ldots \text { end }^{j}\right) \quad K \equiv\left(\ldots\left(K_{i+1}^{k_{i+1}}: K_{i}^{k_{i}}\right) \ldots K_{0}^{k_{0}}\right)}{O \vdash[l c . M, i] \mapsto_{l}\left(\theta, K_{i}^{k_{i}}\right)} \\
& \frac{O \vdash l c \mapsto_{l}\left(\theta,\left(\text { functor }\left(X: A^{j_{1}}\right) \rightarrow K^{j_{2}}\right)^{j_{0}}\right) \quad K \equiv\left(\ldots\left(K_{i+1}^{k_{i+1}}: K_{i}^{k_{i}}\right) \ldots K_{0}^{k_{0}}\right)}{O \vdash[l c(p), i] \mapsto_{l}\left(\theta[X \mapsto p], K_{i}^{k_{i}}\right)} \\
& O \vdash l c \mapsto_{l}\left(\theta \text {, ss } \ldots \text { module } M:=K^{j^{\prime}} \ldots \text { end }^{j}\right) \quad i \geq 1 \\
& \frac{K \equiv\left(\ldots\left(K_{i}^{k_{i}}: K_{i-1}^{k_{i-1}}\right) \ldots K_{0}^{k_{0}}\right) \quad K_{i} \neq\left(J_{1}^{j_{1}}: J_{2}^{j_{2}}\right)}{O \vdash[l c . M, i] \mapsto_{l}\left(\theta, K_{i}^{k_{i}}\right)} \\
& O \vdash l c \mapsto_{l}\left(\theta,\left(\text { functor }\left(X: A^{j_{1}}\right) \rightarrow K^{j_{2}}\right)^{j_{0}}\right) \quad i \geq 1 \\
& \frac{K \equiv\left(\ldots\left(K_{i}^{k_{i}}: K_{i-1}^{k_{i-1}}\right) \ldots K_{0}^{k_{0}}\right) \quad K_{i} \neq\left(J_{1}^{j_{1}}: J_{2}^{j_{2}}\right)}{O \vdash[l c(p), i] \mapsto_{l}\left(\theta[X \mapsto p], K_{i}^{k_{i}}\right)}
\end{aligned}
$$

Figure 27: Locating path look-up with respect to $O$

a module path refers a module relative to the structure or (lazy) structure type to which the path's preceding self variable is ascribed.

The syntax for locating paths is:

$$
l c::=\epsilon|[l c . M, i]|[l c(p), i]
$$

Let us examine each construct. The construct $\epsilon$ represents the top-level. When $l c$ refers to a structure or (lazy) structure type containing a module binding module $M:=$ $K^{j}$, then $[l c . M, i]$ resolves to the module expression or (lazy) signature obtained from $K$ by erasing $K$ 's sealing signatures $i$ times. Similarly, when $l c$ refers to a functor (functor $\left.(X: A) \rightarrow K^{j_{2}}\right)^{j_{1}}$, then $[l c(p), i]$ resolves to the module expression obtained from $K$ by erasing $K$ 's sealing signatures $i$ times. In the constructs $[l c . M, i]$ and $[l c(p), j]$, we call the integers $i$ and $j$ locating integers.

In Figure 27, we define locating path look-up judgment. The judgment $O \vdash l c \mapsto_{l}$ $\left(\theta, K^{i}\right)$ informally means that the locating path $l c$ refers to the module description $K$ labeled with the integer $i$ in the top-level $O$, where each module variable $X$ bound to $\theta(X)$. 


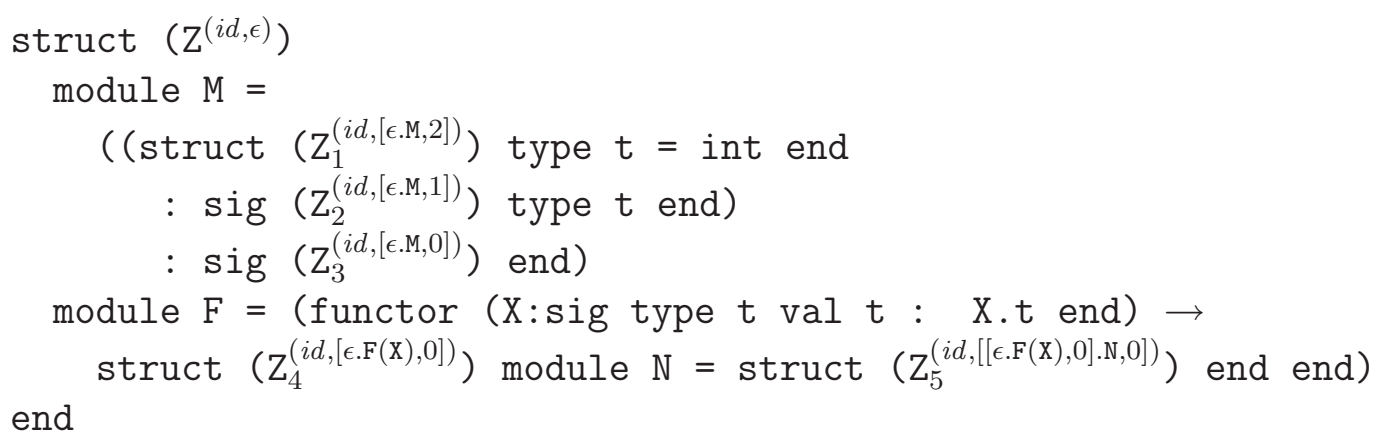

Figure 28: An example for annotations on self variables

$$
\gamma\left(Z^{(\theta, l c)}\right)=l c \quad \gamma(p \cdot M)=[\gamma(p) \cdot M, 0] \quad \gamma\left(p_{1}\left(p_{2}\right)\right)=\left[\gamma\left(p_{1}\right)\left(p_{2}\right), 0\right]
$$

Figure 29: Turning module paths into locating paths

We make the assumption that each occurrence of a self variable $Z$ in a program $P$ is annotated with a locating path $l c$ in addition to an identity substitution, written $Z^{(i d, l c)}$, such that $P \vdash l c \mapsto_{l}\left(i d, \rho_{P}(Z)\right)$ holds. In a practical system, we provide an elaboration phase for complementing these annotations. For an explanatory purpose, we give an example of a program which reflects our assumptions on self variables in Figure 28.

For a module variable environment $\theta$, we define $\theta(l c)$ as follows:

$$
\theta(\epsilon)=\epsilon \quad \theta([l c . M, i])=[\theta(l c) . M, i] \quad \theta([l c(p), i])=[\theta(l c)(\theta(p)), i]
$$

We also define a function $\gamma$ for turning extended module identifiers (i.e., module paths other than module variables) into locating paths in Figure 29. We may say that $\gamma(p)$ is the locating path of $p$. Note that if $O \vdash p \mapsto\left(\theta, K^{i}\right)$ then $O \vdash \gamma(p) \mapsto_{l}\left(\theta, K^{i}\right)$ and vice versa.

Coercible judgments Here, we define coercible judgments for judging whether two types are coercible.

Definition 6 A module path $p$ is in structure form with respect to a top-level $O$ if and only if $O \vdash p \mapsto\left(\theta\right.$, ss ...end $\left.{ }^{i}\right)$ and, for all $X$ in $\operatorname{dom}(\theta), \theta(X)$ is in structure form with respect to $O$.

We define coercible judgments on types and locating paths in Figure 30 and 31, respectively. The judgment $U \vdash \tau::>\tau^{\prime}$ means that the types $\tau$ and $\tau^{\prime}$ are coercible with respect to the lazy program type $U$; the judgment $U \vdash l c::>_{l} l c^{\prime}$ reads similarly. 


$$
\begin{gathered}
\overline{U \vdash \mathbf{1}::>\mathbf{1}} \frac{U \vdash \tau_{11}::>\tau_{21} \quad U \vdash \tau_{12}::>\tau_{22}}{U \vdash \tau_{11} * \tau_{12}::>\tau_{21} * \tau_{22}} \frac{U \vdash \tau_{11}::>\tau_{21} U \vdash \tau_{12}::>\tau_{22}}{U \vdash \tau_{11} \rightarrow \tau_{12}::>\tau_{21} \rightarrow \tau_{22}} \\
\frac{p_{1} \text { and } p_{2} \text { are in structure form with respect to } U \quad U \vdash \gamma\left(p_{1}\right)::>{ }_{l} \gamma\left(p_{2}\right)}{U \vdash p_{1} . t::>p_{2} . t}
\end{gathered}
$$

Figure 30: Convertible relation on types with respect to $U$

$$
\begin{gathered}
\overline{U \vdash \epsilon::>_{l} \epsilon} \overline{U \vdash X::>_{l} X} \frac{U \vdash l c_{1}::>_{l} l c_{2}}{U \vdash\left[l c_{1} \cdot M, i_{1}\right]::>_{l}\left[l c_{2} \cdot M, i_{2}\right]} \\
\frac{U \vdash l c_{1}::>_{l} l_{2} \quad U \vdash \gamma\left(p_{1}\right)::>_{l} \gamma\left(p_{2}\right)}{U \vdash\left[l c_{1}\left(p_{1}\right), i_{1}\right]::>_{l}\left[l c_{2}\left(p_{2}\right), i_{2}\right]}
\end{gathered}
$$

Figure 31: Convertible relation on locations with respect to $U$

For the expression (e: $\tau::>\tau^{\prime}$ ) to be type-correct, two types $\tau$ and $\tau^{\prime}$ must be coercible.

The rules in Figure 30 are mostly straightforward. The last rule says that for two type paths $p_{1} . t$ and $p_{2} . t$ to be coercible they must be in structure form and their locating paths must be coercible. Figure 31 defines the coercible judgment on locating paths. Two locating paths are convertible if and only if they differ only in locating integers.

For instance, consider Figure 1. Assume that the self variable TF of TreeForest is annotated with $(i d, \epsilon)$, and that the module Tree declares a self variable named $\mathrm{T}$, whose annotation would be $(i d,[\epsilon$.Tree, 1$])$. Then the locating path of $\mathrm{TF}^{(i d, \epsilon)}$. Tree is $[\epsilon$. Tree, 0$]$ and that of $\mathrm{T}^{(i d,[\epsilon . \text { Tree,1]) }}$ is $[\epsilon$. Tree, 1$]$. The two locating paths are coercible, hence so are the types $\mathrm{TF}^{(i d, \epsilon)}$.Tree.t and $\mathrm{T}^{(i d,[\epsilon, \operatorname{Tree}, 1])}$.t.

Typing rule In Figure 32, we give a typing rule for type coercion. Observe that when checking coercibility between the types $\tau_{1}$ and $\tau_{2}$, the type system does not expand them.

For conciseness, we usually do not write locating path annotations of self variables.

$$
\begin{gathered}
U \vdash \tau_{1} \diamond \quad U \vdash \tau_{2} \diamond \quad U, \Gamma \vdash e: \tau \quad U \vdash \tau_{1} \equiv \tau \\
U \vdash \tau_{1}::>\tau_{2} \quad \operatorname{Typ} \operatorname{Exp}\left(U, \tau_{2}\right)=\tau_{2}^{\prime}
\end{gathered}
$$

Figure 32: Typing rule for the type coercion with respect to $U$ 


$$
\begin{array}{cl}
\frac{P \vdash p \sim_{n} p^{\prime}}{P \vdash p \cdot M \sim_{n} p^{\prime} \cdot M} & \frac{P \vdash p \sim_{n} p^{\prime}}{P \vdash p(q) \sim_{n} p^{\prime}(q)} \\
\frac{P \vdash q \sim_{n} q^{\prime}}{P \vdash p(q){{ }_{n}}_{n} p\left(q^{\prime}\right)} & \frac{P \vdash p \mapsto_{d}\left(\theta, q^{i}\right)}{P \vdash p \sim_{n} \theta(q)}
\end{array}
$$

Figure 33: Normalization of module paths with respect to $P$

\section{Soundness}

In this section, we present a call-by-value operational semantics and prove a soundness result.

First we define the normalization of module paths in Figure 33. The judgment $P \vdash p \sim_{n} q$ means that $p$ reduces into $q$ in one step, with respect to the program $P$. The normalization traces module abbreviations in the intuitive way, and is defined for module paths containing no module variables.

During normalization and reductions to be defined below, we use implementation look-up judgment instead of the look-up judgment (Figure 7), to look up concrete modules from a program. The judgment is defined in Figure 34, with an auxiliary in Figure 35 .

Values $v$ and evaluation contexts $E$ are:

$$
\begin{aligned}
& v \quad::=()\left|\left(v_{1}, v_{2}\right)\right| \text { p.c } v \mid(\lambda x . e: \tau) \\
& E \quad:=\{\}|(E, e)|(v, E)\left|\pi_{i}(E)\right| E(e)|v(E)| p . c E \\
& \mid \quad \text { case } E \text { of } m s \mid\left(E: \tau_{1}: \tau_{2}\right)
\end{aligned}
$$

where $p$ does not contain module variables.

Then a small step reduction is either:

$$
\begin{gathered}
p . l \stackrel{\text { mp }}{\rightarrow} p^{\prime} . l \quad \text { when } P \vdash p \sim_{n} p^{\prime} \quad \pi_{i}\left(v_{1}, v_{2}\right) \stackrel{\text { prj }}{\rightarrow} v_{i} \quad\left(v: \tau_{1}: \tau_{2}\right) \stackrel{\text { convt }}{\rightarrow} v \\
(\lambda x . e: \tau) v \stackrel{\text { fun }}{\longrightarrow}[x \mapsto v] e \quad \text { case } p . c v \text { of } q . c x \Rightarrow e \stackrel{\text { case }}{\longrightarrow}[x \mapsto v] e \\
p . l \stackrel{\text { vpth }}{\longrightarrow} \theta(e) \text { when } P \vdash p \mapsto_{d}\left(\theta, \text { struct } \ldots \text { val } l=e \ldots \text { end }^{i}\right)
\end{gathered}
$$

or an inner reduction obtained by induction:

$$
\frac{e_{1} \rightarrow e_{2} \quad E \neq\{\}}{E\left\{e_{1}\right\} \rightarrow E\left\{e_{2}\right\}}
$$

Again, these reductions are defined with respect to a program $P$.

When deconstructing a value through the case expression case $p . c v$ of $q . c x$, we do not explicitly check that $p$ and $q$ are equivalent. The type system already ensures that $p$ and $q$ expand into equivalent module paths. 


$$
\begin{gathered}
\overline{P \vdash Z^{\theta} \mapsto_{d}\left(\theta, \rho_{P}(Z)\right)} \\
\frac{P \vdash p \mapsto_{d}\left(\theta, \text { struct } \ldots \text { module } M=E \ldots \text { end }^{i}\right)}{P \vdash p . M \mapsto_{d}(\theta, \text { erase }(E))} \\
\frac{P \vdash p_{1} \mapsto_{d}\left(\theta,(\text { functor }(X: A) \rightarrow E)^{i}\right)}{P \vdash p_{1}\left(p_{2}\right) \mapsto_{d}\left(\theta\left[X \mapsto p_{2}\right], \operatorname{erase}(E)\right)}
\end{gathered}
$$

Figure 34: Implementation look-up

$$
\operatorname{erase}\left((E: S)^{i}\right)=\operatorname{erase}(E) \quad \operatorname{erase}(E)=E \text { otherwise }
$$

Figure 35: Sealing erasure

We assume that the top-level structure of every program $P$ contains a value component named main. The evaluation of $P$ begins by reducing the defining expression of main.

Proposition 6 (Soundness) Let a program $P$ be well-typed. Then the evaluation of $P$ either returns a value or else gives rise to an infinite reduction sequence.

\subsection{Proof of the soundness}

Our proof of Proposition 6 proceeds in the following three steps.

1. We define a type system TraviataY, which uses the intuitive expansion algorithms for expanding module paths and types. We prove that when a program $P$ is type-correct in Traviata, then it is type-correct in TraviataY.

2. We define a type system TraviataX, whose type equivalence relation is defined by the weak bisimulation relation on a labeled transition system on types. We establish a soundness result for TraviataX, by proving progress and subject reduction properties.

3. We prove that if $P$ is type-correct in Traviata $Y$, then it is type-correct in TraviataX.

The purpose of the use of locks $\Sigma, \Omega$ and $\Psi$ during expansions (Figure 9 and 11) and core type reconstruction(Figure 12) is for the decidability result. In the soundness proof, we are interested in a derivation tree which proves well-typedness of $P$ but not in how we can construct the tree. Hence, in the proofs below, we use judgments 
of the ground normalization, the type expansion and the core type reconstruction that do not hold locks. For instance, we may say that " $U \vdash p \sim g q$ holds", when $U, \Sigma \vdash p \sim g q$ can be proven for some $\Sigma$ by the inference rules that are completely same as the rules in Figure 9 but do not have locks. (It is clear that whether or not the inference rules use locks does not affect outputs of the ground normalization; the ground normalization without locks may diverge and the ground normalization with locks may raise more errors than without.)

In the rest of this section, we only consider programs that are well-typed in Traviata, that is, programs $P$ such that $\operatorname{Reconst} P(P)=U_{P}$ and $U_{P} \vdash P: U_{P}$ hold. In particular, we do not explicitly include this premise when stating lemmas. Recall that all core types appearing in $U_{P}$ are located types. This is important for proving Lemma 2. We also assume that all module paths, types and core expressions have located variables. Again, we do not include this premise when stating lemmas and propositions.

\subsubsection{The type system Traviata $Y$}

Traviata $Y$ does not have a lazy program type reconstruction algorithm; it checks that $P$ is type-correct with respect to a given $U_{P}$. Traviata $Y$ differs from Traviata in that it expands module paths and types in the intuitive way.

We define the module path expansion algorithm and the type expansion algorithm that Traviata $Y$ uses in in Figure 36 and Figure 37, respectively. In Figure 39, we present typing rules for TraviataY, with auxiliaries found in Figure 41, 42 and 43. The typing rules are same as those for Traviata, except for the expansion algorithms.

Lemma 1 ([20]) If PathExp $\left(U_{P}, p\right)=q$, then $U_{P} \vdash_{Y} p \leadsto q$.

Lemma 2 If $U_{P} \vdash \tau \downarrow \tau^{\prime}$, then $U_{P} \vdash_{Y} \tau \downarrow \tau^{\prime}$.

Proposition 7 Suppose Reconst $P(P)=U_{P}$ and $U_{P} \vdash P: U_{P}$, then $U_{P} \vdash_{Y} P: U_{P}$.

Proof. By Lemma 1 and 2.

In the rest of proofs, we assume that $\operatorname{Reconst} P(P)=U_{P}$ and $U_{P} \vdash_{Y} P: U_{P}$ hold. In particular, we do not explicitly include this premise when stating lemmas.

For the purpose of proofs, we strengthen the inference rules of well-formedness of module paths (Figure 42), by replacing the rule for self variables with the rule:

$$
\frac{U \vdash_{Y} \theta \mathrm{wf}}{U \vdash_{Y} Z^{\theta} \mathrm{wf}}
$$

where $U \vdash_{Y} \theta$ wf is defined as follows. 
Definition 7 A module variable environment $\theta$ is well-formed with respect to $U$ in Traviata $Y$, written $U \vdash_{Y} \theta$ wf, if and only if, for all $X \in \operatorname{dom}(\theta), U \vdash_{Y} \theta(X)$ wf and $U \vdash_{Y} \theta(X)<\theta\left(\rho_{U}(X)\right)$.

Lemma 3 Let $\theta$ be in located form and $O \vdash_{Y} p \leadsto q$, then $O \vdash_{Y} \theta(p) \leadsto \theta(q)$.

Lemma 4 Let $\theta_{1}$ and $\theta_{2}$ be module variable environments such that $\operatorname{dom}\left(\theta_{1}\right)=$ $\operatorname{dom}\left(\theta_{2}\right)$ and for all $X \in \operatorname{dom}(\theta) O \vdash_{Y} \theta_{1}(X) \leadsto \theta_{2}(X)$. If $O \vdash_{Y} p \leadsto q$, then $O \vdash_{Y} \theta_{1}(p) \leadsto \theta_{2}(q)$.

Lemma 5 Suppose $U_{P} \vdash_{Y} \theta$ wf and $U_{P} \vdash_{Y} p$ wf, then $U_{P} \vdash_{Y} \theta(p)$ wf.

Lemma 6 Suppose $U_{P} \vdash_{Y} \theta$ wf and $U_{P} \vdash_{Y} \tau \diamond$, then $U_{P} \vdash_{Y} \theta(\tau) \diamond$.

Lemma 7 Suppose $U_{P} \vdash_{Y} \theta$ wf and $U_{P}, \Gamma \vdash_{Y} e: \tau$. Then $U_{P}, \Gamma_{1} \vdash_{Y} \theta(e): \tau^{\prime}$ with $U_{P} \vdash_{Y} \theta(\tau) \equiv \tau^{\prime}$, where $\operatorname{dom}(\Gamma)=\operatorname{dom}\left(\Gamma_{1}\right)$ and, for all $x$ in $\operatorname{dom}\left(\Gamma_{1}\right), U_{P} \vdash_{Y}$ $\theta(\Gamma(x)) \equiv \Gamma_{1}(x)$.

\subsubsection{The type system TraviataX}

Before presenting Traviata $X$, we build a lazy program type $U_{P}^{\sharp}$ from $U_{P}$ by making all abstract type specifications except for those appearing in module variable signatures transparent. We define a function Trans in Figure 44, which builds $U_{P}^{\sharp}$ from $U_{P}$. We let $U_{P}^{\sharp}$ be $\operatorname{Trans}\left(U_{P}\right)$. Note that bound self variables may escape their scope in $U_{P}^{\sharp}$.

TraviataX differs from Traviata $Y$ in that a type equivalence relation of TraviataX is defined by the weak bisumilation relation on a labeled transition system on types.

We define labeled transition systems on module paths and types in Figure 46 and 47 , respectively. Note that, in $U_{P}^{\sharp}$, abstract type specifications may only appear in module variable signatures.

We write $U \vdash \tau \stackrel{c}{\Rightarrow} \tau^{\prime}$ to mean $U \vdash \tau \Rightarrow \stackrel{c}{\Rightarrow} \Rightarrow \tau^{\prime}$, where $\Rightarrow$ is the reflective transitive closure of $\stackrel{\tau}{\longrightarrow}$. (We use juxtaposition to denote relation composition.)

Definition 8 ([18]) Let $\mathcal{R}$ be a binary relation on types. Then $\mathcal{R}$ is a weak simulation with respect to a lazy program type $U$ if, whenever $\tau_{1} \mathcal{R} \tau_{2}$,

1. if $U \vdash \tau_{1} \stackrel{\tau}{\rightarrow} \tau_{1}^{\prime}$ then there exists $\tau_{2}^{\prime}$ such that $U \vdash \tau_{2} \Rightarrow \tau_{2}^{\prime}$ and $\tau_{1}^{\prime} \mathcal{R} \tau_{2}^{\prime}$.

2. if $U \vdash \tau_{1} \stackrel{c}{\rightarrow} \tau_{1}^{\prime}$ then there exists $\tau_{2}^{\prime}$ such that $U \vdash \tau_{2} \stackrel{c}{\Rightarrow} \tau_{2}^{\prime}$ and $\tau_{1}^{\prime} \mathcal{R} \tau_{2}^{\prime}$. 
Definition 9 ([18]) A binary relation $\mathcal{R}$ on types is said to be a weak bisimulation with respect to a lazy program type $U$ if both $\mathcal{R}$ and its converse are weak simulations with respect to $U$. We say that $\tau$ and $\tau^{\prime}$ are weakly bisimilar with respect to $U$, written $U \vdash \tau \approx \tau^{\prime}$, if there exists a weak bisimulation $\mathcal{R}$ with respect to $U$ such that $\tau \mathcal{R} \tau^{\prime}$.

Again, we usually write $\tau \approx \tau^{\prime}$ to denote $U_{P}^{\sharp} \vdash \tau \approx \tau^{\prime}$.

Lemma $8([\mathbf{1 8}])$ The relation $\approx$ is a weak bisimulation.

We present typing rules for TraviataX in Figure 49, with auxiliaries in Figure 50, 51 and 52 .

Definition 10 A program $P$ is type-correct with respect to $U$ in TraviataX if and only if $U \vdash_{X} P: U$ holds.

For the purpose of proofs, we strengthen the inference rules of well-formedness of module paths (Figure 51), by replacing the rule for self variables with the rule:

$$
\frac{U \vdash_{X} \theta \text { wf }}{U \vdash_{X} Z^{\theta} \text { wf }}
$$

where $U \vdash_{X} \theta$ wf is defined as follows.

Definition 11 A module variable environment $\theta$ is well-formed with respect to a lazy program type $U$ in TraviataX, written $U \vdash_{X} \theta$ wf, if and only if, for all $X \in \operatorname{dom}(\theta)$, $U \vdash_{X} \theta(X)$ wf and $U \vdash_{X} \theta(X)<\theta\left(\rho_{U}(X)\right)$.

Definition 12 For types $\tau$ and $\tau^{\prime}$, we write $U \vdash \tau \stackrel{\tau^{*}}{\leftrightarrow} \tau^{\prime}$ when $\left(\tau, \tau^{\prime}\right)$ is an element of the smallest transitive and symmetric relation containing $\left\{\left(\tau, \tau^{\prime}\right) \mid U \vdash \tau \stackrel{\tau}{\rightarrow} \tau^{\prime}\right\}$.

Lemma 9 If $U_{P}^{\sharp} \vdash \tau \stackrel{c}{\rightarrow} \tau_{1}$ and either $U_{P}^{\sharp} \vdash \tau \stackrel{\tau}{\rightarrow} \tau^{\prime}$ or $U_{P}^{\sharp} \vdash \tau^{\prime} \stackrel{\tau}{\longrightarrow} \tau$, then $U_{P}^{\sharp} \vdash \tau^{\prime} \stackrel{c}{\Rightarrow}$ $\tau_{2}$ and $U_{P}^{\sharp} \vdash \tau_{1} \stackrel{\tau^{*}}{\leftrightarrow} \tau_{2}$.

Lemma 10 If $U_{P}^{\sharp} \vdash \tau_{0} \stackrel{\tau}{\longrightarrow} \tau_{1}$ and $U_{P}^{\sharp} \vdash \tau_{0} \stackrel{c}{\Rightarrow} \tau_{2}$, then $U_{P}^{\sharp} \vdash \tau_{1} \stackrel{c}{\Rightarrow} \tau_{3}$ and $U_{P}^{\sharp} \vdash \tau_{2} \stackrel{\tau^{*}}{\leftrightarrow}$ $\tau_{3}$.

Lemma 11 If $U_{P}^{\sharp} \vdash \tau \stackrel{\tau^{*}}{\leftrightarrow} \tau^{\prime}$ and $U_{P}^{\sharp} \vdash \tau \stackrel{c}{\hookrightarrow} \tau_{1}$, then $U_{P}^{\sharp} \vdash \tau^{\prime} \stackrel{c}{\Rightarrow} \tau_{2}$ and $U_{P}^{\sharp} \vdash \tau_{1} \stackrel{\tau^{*}}{\leftrightarrow} \tau_{2}$.

The following lemma is important; it says that $\tau$-transitions preserve bisimilarity. In particular, using this lemma we prove Lemma 16. 
Lemma 12 If $U_{P}^{\sharp} \vdash \tau \stackrel{\tau}{\rightarrow} \tau^{\prime}$, then $U_{P}^{\sharp} \vdash \tau \approx \tau^{\prime}$.

Proof. Prove and use Lemmas 9 to 11.

Lemma 13 If $U_{P} \vdash_{Y} p \leadsto p^{\prime}$, then $U_{P}^{\sharp} \vdash p \Rightarrow p^{\prime}$.

Lemma 14 If $U_{P} \vdash_{Y} \tau \downarrow \tau^{\prime}$, then $U_{P}^{\sharp} \vdash \tau \approx \tau^{\prime}$.

Lemma 15 If $U_{P} \vdash \tau \equiv_{\tau} \tau^{\prime}$, then $U_{P}^{\sharp} \vdash \tau \approx \tau^{\prime}$.

The following lemma is important; it says that type equivalence in TraviataY implies that in TraviataX.

Lemma 16 If $U_{P} \vdash_{Y} \tau \equiv \tau^{\prime}$, then $U_{P}^{\sharp} \vdash \tau \approx \tau^{\prime}$

Lemma 17 If $U_{P} \vdash_{Y} p$ wf and $U_{P}^{\sharp} \vdash p \stackrel{\tau}{\longrightarrow} p^{\prime}$, then $U_{P} \vdash_{Y} p^{\prime}$ wf.

Proof. Use Lemma 5.

Lemma 18 If $U_{P} \vdash_{Y} \tau \diamond$ and $U_{P} \vdash \tau \stackrel{\tau}{\rightarrow} \tau^{\prime}$, then $U_{P} \vdash_{Y} \tau^{\prime} \diamond$.

Proof. Use Lemma 6.

Lemma 19 If $U_{P}^{\sharp}, \Gamma \vdash_{X} e: \tau_{1}$ and $U_{P}^{\sharp}, \Gamma \vdash_{X} e: \tau_{2}$, then $U_{P}^{\sharp} \vdash \tau_{1} \approx \tau_{2}$

Lemma 20 Let a relation $\mathcal{R}$ on types be defined as:

$$
\mathcal{R} \stackrel{\text { def }}{=}\left\{\left(\theta(\tau), \theta\left(\tau^{\prime}\right)\right) \mid U_{P} \vdash_{Y} \theta \text { wf and } U_{P}^{\sharp} \vdash \tau \approx \tau^{\prime}\right\}
$$

If $\left(\tau, \tau^{\prime}\right) \in \mathcal{R}$, then $U_{P}^{\sharp} \vdash \tau \approx \tau^{\prime}$.

Lemma 21 If $U_{P} \vdash_{Y} p$ wf, then $U_{P}^{\sharp} \vdash_{X} p$ wf.

Proof. Use Lemma 20.

Lemma 22 If $U_{P} \vdash_{Y} \tau \diamond$, then $U_{P}^{\sharp} \vdash_{X} \tau \diamond$.

Proof. Use Lemma 21. 
Lemma 23 Let $U_{P}^{\sharp} \vdash_{X} \theta$ wf and $\theta$ is in located form. Let a relation $\mathcal{R}$ on types be defined as:

$$
\mathcal{R} \stackrel{\text { def }}{=}\left\{\left(\theta(\tau), \theta\left(\tau^{\prime}\right)\right) \mid U_{P}^{\sharp} \vdash \tau \approx \tau^{\prime}\right\}
$$

If $\left(\tau, \tau^{\prime}\right) \in \mathcal{R}$, then $U_{P}^{\sharp} \vdash \tau \approx \tau^{\prime}$.

Lemma 24 If $U_{P}^{\sharp} \vdash_{X} \theta$ wf and $\theta$ is in located form and $U_{P}^{\sharp} \vdash_{X} p$ wf, then $U_{P}^{\sharp} \vdash_{X}$ $\theta(p) \mathrm{wf}$.

Lemma 25 Let $U_{P}^{\sharp} \vdash_{X} p$ wf and $U_{P}^{\sharp} \vdash_{Y} p \leadsto q$, then $U_{P}^{\sharp} \vdash_{X} q$ wf.

Lemma 26 Let $U_{P}^{\sharp} \vdash_{X} \theta$ wf and $U_{P}^{\sharp} \vdash_{X} p$ wf, then $U_{P}^{\sharp} \vdash_{X} \theta(p)$ wf.

Lemma 27 Let $U_{P}^{\sharp} \vdash_{X} p$ wf and $U_{P}^{\sharp} \vdash p \stackrel{\tau}{\longrightarrow} p^{\prime}$, then $U_{P}^{\sharp} \vdash_{X} p^{\prime}$ wf.

Lemma 28 Let a relation $\mathcal{R}$ on types be defined as:

$$
\mathcal{R} \stackrel{\text { def }}{=}\left\{\left(\theta(\tau), \theta\left(\tau^{\prime}\right)\right) \mid U_{P}^{\sharp} \vdash_{X} \theta \text { wf and } U_{P}^{\sharp} \vdash \tau \approx \tau^{\prime}\right\}
$$

If $\left(\tau, \tau^{\prime}\right) \in \mathcal{R}$, then $U_{P}^{\sharp} \vdash \tau \approx \tau^{\prime}$.

Lemma 29 Let $U_{P}^{\sharp} \vdash_{X} \theta$ wf and $U_{P}^{\sharp}, \Gamma \vdash_{X} e: \tau$, then $U_{P}^{\sharp}, \Gamma_{1} \vdash_{X} \theta(e): \theta(\tau)$, where $\operatorname{dom}(\Gamma)=\operatorname{dom}\left(\Gamma_{1}\right)$, and for all $x \in \operatorname{dom}(\Gamma) U_{P}^{\sharp} \vdash \Gamma_{1}(x) \approx \theta(\Gamma(x))$.

We build a mapping from self variables to module paths, using functions defined in Figure 45. We let $\mathcal{S}$ be $\operatorname{SelfMap}\left(U_{P}\right)$. Intuitively, $\mathcal{S}$ corrects substitutions of module paths for self variables which are performed by Traviata $Y$ during type checking of $P$ for ensuring that sealing expressions $(E: S)$ are type-correct.

For a type $\tau$, we write $\tau^{\mathcal{S}}$ to denote the set of types obtained from $\tau$ by replacing some occurrences of $Z^{\theta}$ in $\tau$ with $\theta(\mathcal{S}(Z))$. We use $\tau^{s}, \tau^{s_{1}}, \tau^{s_{2}}$ and the likes as metavariables for elements of $\tau^{\mathcal{S}}$. We also write $p^{\mathcal{S}}$ and $p^{s}$ with obvious meanings.

Observe that, for all $Z \in \operatorname{dom}(\mathcal{S})$, we have $U_{P}^{\sharp} \vdash_{X} \mathcal{S}(Z)<T_{d}$, where $U_{P}^{\sharp} \vdash Z^{\text {id }} \mapsto$ $\left(i d, T_{d}^{i}\right)$.

We define a binary relation $\approx_{\mathcal{S}}$ on types as follows:

$$
\approx_{\mathcal{S}}=\left\{\left(\tau, \tau^{s}\right) \mid U_{P}^{\sharp} \vdash_{X} \tau \text { wf and } \tau^{s} \in \tau^{\mathcal{S}}\right\} .
$$

Lemma 30 If $U_{P}^{\sharp} \vdash \tau \approx_{\mathcal{S}} \tau^{\prime}$ then $U_{P}^{\sharp} \vdash \tau \approx \tau^{\prime}$. 
Lemma 31 If $U_{P}^{\sharp} \vdash_{X} \tau \diamond$ and $U_{P}^{\sharp} \vdash_{X} \tau^{\prime} \diamond$ and $U_{P}^{\sharp} \vdash \tau::>\tau^{\prime}$, then $U_{P}^{\sharp} \vdash \tau \approx \tau^{\prime}$.

Lemma 32 If $U_{P}, \Gamma \vdash_{Y} e: \tau$, then $U_{P}^{\sharp}, \Gamma \vdash_{X} e: \tau$.

Proposition 8 Assume Reconst $P(P)=U_{P}$ and $U_{P} \vdash_{Y} P: U_{P}$ and $\operatorname{Trans}\left(U_{P}\right)=$ $U_{P}^{\sharp}$, then $U_{P}^{\sharp} \vdash_{X} P: U_{P}^{\sharp}$.

Proposition 9 (Subject reduction in TraviataX) Assume Reconst $P(P)=U_{P}$ and $U_{P} \vdash_{Y} P: U_{P}$ and $\operatorname{Trans}\left(U_{P}\right)=U_{P}^{\sharp}$, and $U_{P}^{\sharp} \vdash_{X} P: U_{P}^{\sharp}$. If $U_{P}^{\sharp}, \emptyset \vdash_{X} e: \tau_{1}$ and $e \rightarrow e^{\prime}$, then $U_{P}^{\sharp}, \emptyset \vdash_{X} e^{\prime}: \tau_{1}$.

Definition 13 A type $\tau$ is stable with respect to a lazy program type $U$ if and only if, for all $\tau^{\prime}$ in comps $(\tau), \tau^{\prime}$ is a type path p.t where $p$ does not contain module variables and $U_{P}^{\sharp} \vdash p \mapsto\left(\theta\right.$, sig ... datatype $t=c$ of $\tau^{\prime \prime} \ldots$ end $\left.{ }^{i}\right)$ holds.

The function comps on types is defined as follows:

$$
\operatorname{comps}(\tau)= \begin{cases}\emptyset & \tau=\tau_{1} \rightarrow \tau_{2} \text { or } \tau=\mathbf{1} \\ \operatorname{comps}\left(\tau_{1}\right) \cup \operatorname{comps}\left(\tau_{2}\right) & \tau=\tau_{1} * \tau_{2} \\ \{p . t\} & \tau=p . t\end{cases}
$$

Lemma 33 If $U_{P}^{\sharp}, \emptyset \vdash_{X} v: \tau$, then there is a stable type $\tau^{\prime}$ with respect to $U_{P}^{\sharp}$ such that $U_{P}^{\sharp}, \emptyset \vdash_{X} v: \tau^{\prime}$.

\section{Lemma 34}

1. There are no types $\tau_{1}, \tau_{2}$ such that either $U_{P}^{\sharp}, \Gamma \vdash_{X}(): \tau_{1} * \tau_{2}$ or $U_{P}^{\sharp}, \Gamma \vdash_{X}()$ : $\tau_{1} \rightarrow \tau_{2}$ holds.

2. There is not a stable type p.t such that $U_{P}^{\sharp}, \Gamma \vdash_{X}()$ : p.t.

3. If $U_{P}^{\sharp}, \Gamma \vdash_{X}\left(v_{1}, v_{2}\right): \tau$, then there are no types $\tau_{1}, \tau_{2}$ such that $U_{P}^{\sharp}, \Gamma \vdash_{X}$ $\left(v_{1}, v_{2}\right): \tau_{1} \rightarrow \tau_{2}$.

4. If $U_{P}^{\sharp}, \Gamma \vdash_{X}\left(v_{1}, v_{2}\right): \tau$, then $U_{P}^{\sharp}, \Gamma \vdash_{X}\left(v_{1}, v_{2}\right): \mathbf{1}$ does not hold.

5. If $U_{P}^{\sharp}, \Gamma \vdash_{X}\left(v_{1}, v_{2}\right): \tau$, then there is no stable type p.t such that $U_{P}^{\sharp}, \Gamma \vdash_{X}$ $\left(v_{1}, v_{2}\right)$ : p.t.

6. If $U_{P}^{\sharp}, \Gamma \vdash_{X}(\lambda x . e: \tau): \tau^{\prime}$, then there are no types $\tau_{1}, \tau_{2}$ such that $U_{P}^{\sharp}, \Gamma \vdash_{X}$ $(\lambda x . e: \tau): \tau_{1} * \tau_{2}$. 
7. If $U_{P}^{\sharp}, \Gamma \vdash_{X}(\lambda x . e: \tau): \tau^{\prime}$, then $U_{P}^{\sharp}, \Gamma \vdash_{X}(\lambda x . e: \tau): \mathbf{1}$ does not holds.

8. If $U_{P}^{\sharp}, \Gamma \vdash_{X}(\lambda x . e: \tau): \tau^{\prime}$, then there is no stable type p.t such that $U_{P}^{\sharp}, \Gamma \vdash_{X}$ $(\lambda x . e: \tau): p . t$.

9. If $U_{P}^{\sharp}, \Gamma \vdash_{X}$ p.c $v: \tau$, then there are no types $\tau_{1}, \tau_{2}$ such that either $U_{P}^{\sharp}, \Gamma \vdash_{X}$ p.c $v: \tau_{1} \rightarrow \tau_{2}$ or $U_{P}^{\sharp}, \Gamma \vdash_{X}$ p.c $v: \tau_{1} * \tau_{2}$ holds.

10. If $U_{P}^{\sharp}, \Gamma \vdash_{X}$ p.c $v: \tau$, then $U_{P}^{\sharp}, \Gamma \vdash_{X}$ p.c $v: \mathbf{1}$ does not hold.

Proposition 10 (Progress in TraviataX) Assume Reconst $P(P)=U_{P}$ and $U_{P} \vdash_{Y}$ $P: U_{P}$ and Trans $\left(U_{P}\right)=U_{P}^{\sharp}$, and $U_{P}^{\sharp} \vdash_{X} P: U_{P}^{\sharp}$. If $U_{P}^{\sharp}, \emptyset \vdash_{X} e: \tau$ then either $e$ is a value or else there is some $e^{\prime}$ with $e \rightarrow e^{\prime}$ with respect to $P$. 


$$
\begin{array}{cc}
\overline{O \vdash_{Y} X \leadsto X} & \overline{O \vdash_{Y} Z^{\theta} \leadsto Z^{\theta}} \\
O \vdash_{Y} p \leadsto p^{\prime} & O \vdash_{Y} p \leadsto p^{\prime} \\
\frac{O \vdash p^{\prime} . M \mapsto^{i}\left(\theta, K^{i}\right)}{O \vdash_{Y} p \cdot M \leadsto p^{\prime} \cdot M} & \frac{O \vdash p^{\prime} \cdot M \mapsto\left(\theta, q^{i}\right) \quad O \vdash_{Y} \theta(q) \sim r}{O \vdash_{Y} p \cdot M \sim r} \\
O \vdash_{Y} p_{1} \leadsto p_{1}^{\prime} \quad O \vdash_{Y} p_{2} \leadsto p_{2}^{\prime} & O \vdash_{Y} p_{1} \leadsto p_{1}^{\prime} \quad O \vdash_{Y} p_{2} \leadsto p_{2}^{\prime} \\
\frac{O \vdash_{1}^{\prime}\left(p_{2}^{\prime}\right) \mapsto_{\left(\theta, K^{i}\right)} K \neq q}{O \vdash_{Y} p_{1}\left(p_{2}\right) \leadsto p_{1}^{\prime}\left(p_{2}^{\prime}\right)} & \frac{O \vdash_{1}^{\prime}\left(p_{2}^{\prime}\right) \mapsto\left(\theta, q^{i}\right) \quad O \vdash_{Y} \theta(q) \sim r}{O \vdash_{Y} p_{1}\left(p_{2}\right) \leadsto r}
\end{array}
$$

Figure 36: Module path expansion with respect to $O$ in Traviata $Y$

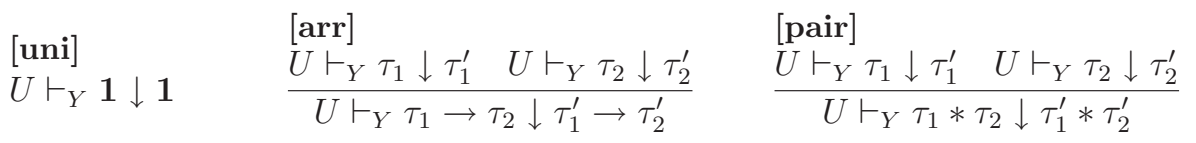

$$
\begin{aligned}
& \frac{\left[\begin{array}{l}
\text { dtyp] } \\
U \vdash_{Y} p \leadsto p^{\prime} \quad U \vdash p^{\prime} . t \mapsto\left(\theta, \text { sig } \ldots \text { datatype } t=c \text { of } \tau \ldots \text { end }{ }^{i}\right)
\end{array}\right.}{U \vdash_{Y} p . t \downarrow p^{\prime} . t} \\
& \text { [opq] } \\
& \frac{U \vdash_{Y} p \leadsto p^{\prime} \quad U \vdash p^{\prime} . t \mapsto\left(\theta, \text { sig } \ldots \text { type } t \ldots \text { end } d^{i}\right)}{U \vdash_{Y} p . t \downarrow p^{\prime} . t}
\end{aligned}
$$

Figure 37: Type expansion with respect to $U$ in Traviata $Y$

$$
\frac{U \vdash_{Y} \tau_{1} \downarrow \tau_{1}^{\prime} \quad U \vdash_{Y} \tau_{2} \downarrow \tau_{2}^{\prime} \quad U \vdash \tau_{1}^{\prime} \equiv_{\tau} \tau_{2}^{\prime}}{U \vdash_{Y} \tau_{1} \equiv \tau_{2}}
$$

Figure 38: Type equivalence with respect to $U$ in Traviata $Y$ 
Definitions and Specifications

$$
\begin{gathered}
\frac{U \vdash_{Y} E: T}{U \vdash_{Y} \text { module } M=E: \text { module } M: T} \quad \frac{U \vdash_{Y} S: T}{U \vdash_{Y} \text { module } M: S: \text { module } M: T} \\
\frac{U \vdash_{Y} \tau_{1} \diamond \quad U \vdash_{Y} \tau_{1} \downarrow \tau_{2}}{U \vdash_{Y} \text { datatype } t=c \text { of } \tau_{1}: \text { datatype } t=c \text { of } \tau_{2}} \\
\frac{U \vdash_{Y} \tau_{1} \diamond U \vdash_{Y} \tau_{1} \downarrow \tau_{2}}{U \vdash_{Y} \text { type } t=\tau_{1}: \operatorname{type} t=\tau_{2}} \quad \frac{\bar{U} \vdash_{Y} \text { type } t: \text { type } t}{U, \emptyset \vdash_{Y} e: \tau} \quad \frac{U \vdash_{Y} \tau_{1} \diamond U \vdash_{Y} \tau_{1} \downarrow \tau_{2}}{U \vdash_{Y} \operatorname{val} l: \tau_{1}: \operatorname{val} l: \tau_{2}} \\
\frac{U \vdash_{Y} \operatorname{val} l=e: \operatorname{val} l: \tau}{\text { Module expression }} \\
\frac{U \vdash_{Y} E_{d}: T_{d}}{U \vdash_{Y} E_{d}^{i}: T_{d}^{i}}
\end{gathered}
$$

Module expression descriptions

$$
\begin{gathered}
\frac{U \vdash_{Y} D_{1}: C_{1} \ldots U \vdash_{Y} D_{n}: C_{n}}{U \vdash_{Y} \text { struct }(Z) D_{1} \ldots D_{n} \text { end }: \operatorname{sig}(Z) C_{1} \ldots C_{n} \text { end }} \\
\frac{U \vdash_{Y} A: A^{\prime} \quad U \vdash_{Y} E: T}{U \vdash_{Y} \text { functor }(X: A) \rightarrow E: \text { functor }\left(X: A^{\prime}\right) \rightarrow T} \\
\frac{U \vdash_{Y} E: T_{1} \quad U \vdash_{Y} S: T_{2} \quad U \vdash_{Y} T_{1}<\operatorname{Subst}\left(T_{1}, S\right)}{U \vdash_{Y}(E: S):\left(T_{1}: T_{2}\right)} \frac{U \vdash_{Y} p \text { wf }}{U \vdash_{Y} p: p} \\
\text { Signature } \\
\frac{U \vdash_{Y} S_{d}: T_{d}}{U \vdash_{Y} S_{d}^{i}: T_{d}^{i}}
\end{gathered}
$$

Signature descriptions

$$
\begin{gathered}
\frac{U \vdash_{Y} B_{1}: C_{1} \ldots U \vdash_{Y} B_{n}: C_{n}}{U \vdash_{Y} \operatorname{sig}(Z) B_{1} \ldots B_{n} \text { end }: \operatorname{sig}(Z) C_{1} \ldots C_{n} \text { end }} \\
\frac{U \vdash_{Y} A: A^{\prime} \quad U \vdash_{Y} S: T}{U \vdash_{Y} \text { functor }(X: A) \rightarrow S: \text { functor }\left(X: A^{\prime}\right) \rightarrow T}
\end{gathered}
$$

Module variable signature

$$
\frac{U \vdash_{Y} A_{d 1}: A_{d 2}}{U \vdash_{Y} A_{d 1}^{i_{1}}: A_{d 2}^{i_{2}}}
$$

Module variable signature description

$$
\frac{U \vdash_{Y} B_{1}: B_{1}^{\prime} \ldots U \vdash_{Y} B_{n}: B_{n}^{\prime}}{U \vdash_{Y} \operatorname{sig} B_{1} \ldots B_{n} \text { end }: \operatorname{sig} B_{1}^{\prime} \ldots B_{n}^{\prime} \text { end }}
$$

Figure 39: Typing rules for the module language with respect to $U$ in Traviata $Y$ 


$$
\begin{aligned}
& \text { Core types } \\
& \overline{U \vdash_{Y} \mathbf{1} \diamond} \\
& \frac{U \vdash_{Y} \tau_{1} \diamond U \vdash_{Y} \tau_{2} \diamond}{U \vdash_{Y} \tau_{1} \rightarrow \tau_{2} \diamond} \\
& \frac{U \vdash_{Y} \tau_{1} \diamond U \vdash_{Y} \tau_{2} \diamond}{U \vdash_{Y} \tau_{1} * \tau_{2} \diamond} \quad \frac{U_{P} \vdash_{Y} p \text { wf } U \vdash_{Y} p . t \downarrow \tau}{U \vdash_{Y} p . t \diamond} \\
& \text { Core expressions }
\end{aligned}
$$

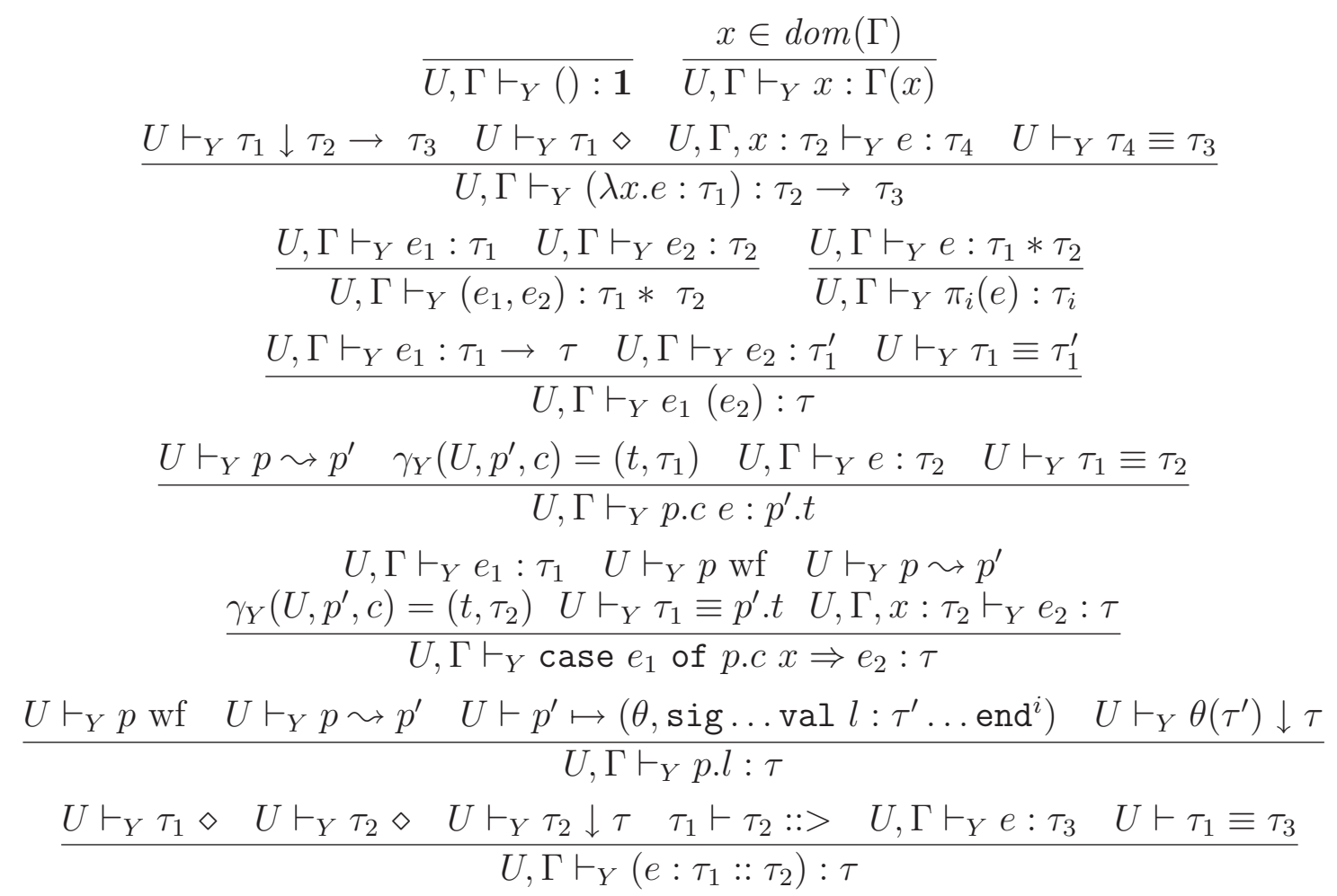

Figure 40: Typing rules for the core language with respect to $U$ in Traviata $Y$

$\gamma_{Y}(U, p, c)=(t, \tau)$ when

$U \vdash p \mapsto\left(\theta\right.$, sig ... datatype $t=c$ of $\tau^{\prime} \ldots$ end $\left.{ }^{i}\right)$ and $U \vdash_{Y} \theta\left(\tau^{\prime}\right) \downarrow \tau$

Figure 41: Datatype look-up with respect to $U$ in Traviata $Y$ 


$$
\begin{aligned}
& \overline{U \vdash_{Y} X \mathrm{wf}} \quad \overline{U \vdash_{Y} Z^{i d} \mathrm{wf}} \quad \frac{U \vdash_{Y} p \cdot M \leadsto q U \vdash_{Y} p \mathrm{wf}}{U \vdash_{Y} p \cdot M \mathrm{wf}} \\
& U \vdash_{Y} p_{1} \text { wf } U \vdash_{Y} p_{2} \text { wf } \quad U \vdash_{Y} p_{1} \leadsto p_{1}^{\prime} \quad U \vdash_{Y} p_{2} \leadsto p_{2}^{\prime} \\
& \frac{U \vdash_{Y} p_{1}\left(p_{2}\right) \leadsto q \quad U \vdash p_{1}^{\prime} \mapsto\left(\theta,\left(\text { functor }\left(X: A_{d}^{j}\right) \rightarrow T\right)^{i}\right) \quad U \vdash_{Y} p_{2}^{\prime}<\theta\left[X \mapsto p_{2}^{\prime}\right]\left(A_{d}\right)}{U \vdash_{Y} p_{1}\left(p_{2}\right) \mathrm{wf}}
\end{aligned}
$$

Figure 42: Well-formed module paths with respect to $U$ in Traviata $Y$

$$
\begin{gathered}
\frac{U \vdash_{Y} T_{d}<S_{d}}{U \vdash_{Y} T_{d}^{i}<S_{d}^{j}} \quad \frac{U \vdash_{Y} T_{d}<A_{d}}{U \vdash_{Y} T_{d}^{i}<A_{d}^{j}} \\
\frac{U \vdash_{Y} T_{d}<S_{d}}{U \vdash_{Y}\left(T: T_{d}^{i}\right)<S_{d}} \\
\frac{U \vdash_{Y} p \leadsto p^{\prime} \quad U \vdash p^{\prime} \mapsto\left(\theta, T_{d}^{i}\right) \quad U \vdash_{Y} \theta\left(T_{d}\right)<S_{d}}{U:\{1, \ldots, m\} \mapsto\{1, \ldots, n\} \quad \forall i \in\{1, \ldots, m\}, U \vdash_{Y} C_{\sigma(i)}<B_{i}} \\
\frac{U \vdash_{Y} \operatorname{sig}(Z) C_{1} \ldots C_{n} \text { end }<\operatorname{sig}\left(Z^{\prime}\right) B_{1} \ldots B_{m} \text { end }}{U \vdash_{Y} A^{\prime}<A \quad U \vdash_{Y}\left[X \mapsto X^{\prime}\right] T<S} \\
\frac{\sigma:\{1, \ldots, m\} \mapsto\{1, \ldots, n\} \quad \forall i \in\{1, \ldots, m\}, U \vdash_{Y} C_{\sigma(i)}<B_{i}}{U \vdash_{Y} \text { functor }(X: A) \rightarrow T<\text { functor }\left(X^{\prime}: A^{\prime}\right) \rightarrow S}
\end{gathered}
$$

$\overline{U \vdash_{Y} \text { type } t<\text { type } t} \quad \overline{U \vdash_{Y} \text { type } t=\tau<\text { type } t} \quad \overline{U \vdash_{Y} \text { datatype } t=c \text { of } \tau<\text { type } t}$

$$
\begin{aligned}
& \frac{U \vdash_{Y} \tau_{1} \equiv \tau_{2}}{U \vdash_{Y} \text { type } t=\tau_{1}<\text { type } t=\tau_{2}} \quad \frac{U \vdash_{Y} \tau_{1} \equiv \tau_{2}}{U \vdash_{Y} \operatorname{val} l: \tau_{1}<\operatorname{val} l: \tau_{2}} \\
& \frac{U \vdash_{Y} \tau_{1} \equiv \tau_{2}}{U \vdash_{Y} \text { datatype } t=c \text { of } \tau_{1}<\text { datatype } t=c \text { of } \tau_{2}} \\
& U \vdash_{Y} T<S \\
& \bar{U} \vdash_{Y} \text { module } M: T<\text { module } M: S
\end{aligned}
$$

Figure 43: Subtyping with respect to $U$ in TraviataY 


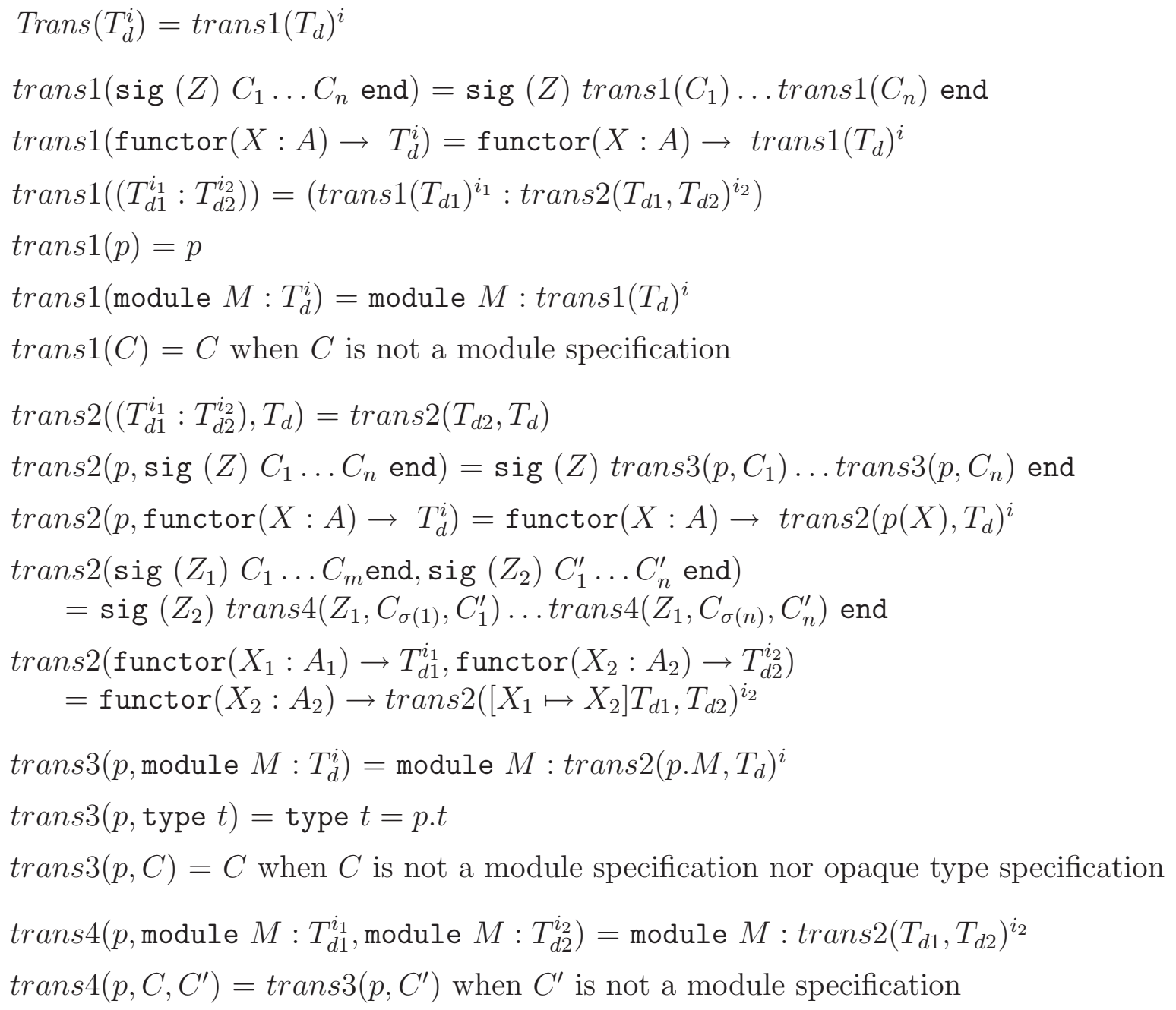

Figure 44: Breaking type abstraction 


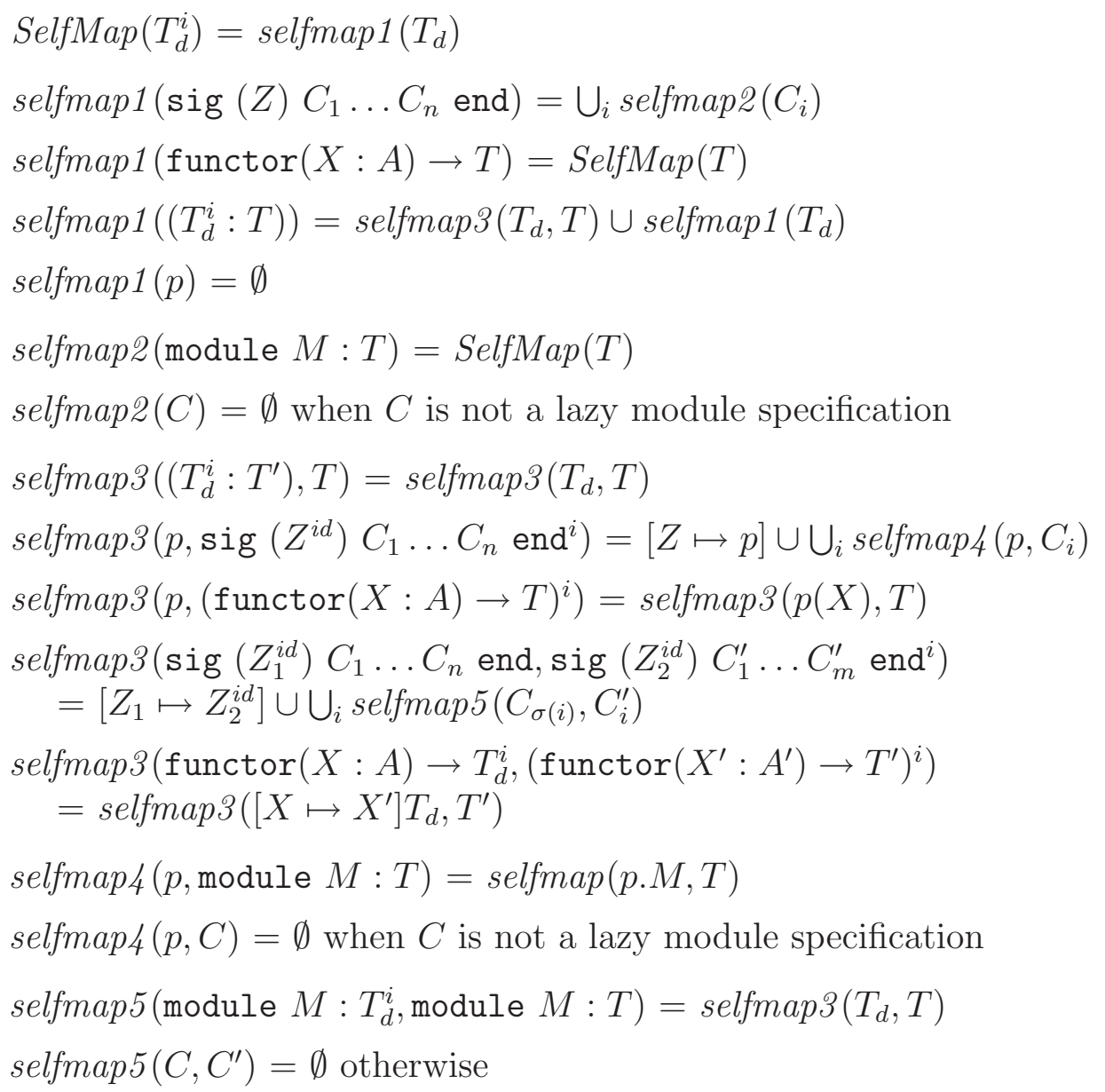

Figure 45: Extracting substitutions 


$$
\begin{gathered}
\operatorname{dom}\left(\theta_{1}\right)=\operatorname{dom}\left(\theta_{2}\right) \quad \exists X \in \operatorname{dom}\left(\theta_{1}\right), U \vdash \theta_{1}(X) \stackrel{\tau}{\rightarrow} \theta_{2}(X) \\
\forall X^{\prime} \in \operatorname{dom}\left(\theta_{1}\right) \backslash\{X\}, \theta_{1}\left(X^{\prime}\right)=\theta_{2}\left(X^{\prime}\right) \\
U \vdash Z^{\theta_{1}} \stackrel{\tau}{\sim} Z^{\theta_{2}} \\
\frac{U \vdash p \stackrel{\tau}{\sim} p^{\prime}}{U \vdash p \cdot M \stackrel{\tau}{\rightarrow} p^{\prime} \cdot M} \quad \frac{U \vdash p \stackrel{\tau}{\sim} p^{\prime}}{U \vdash p(q) \stackrel{\tau}{\rightarrow} p^{\prime}(q)} \\
\frac{U \vdash q \stackrel{\tau}{\rightarrow} q^{\prime}}{U \vdash p(q) \stackrel{\tau}{\longrightarrow} p\left(q^{\prime}\right)} \quad \frac{U \vdash p \mapsto\left(\theta, q^{i}\right)}{U \vdash p \stackrel{\tau}{\sim} \theta(q)}
\end{gathered}
$$

Figure 46: Transition rules for paths with respect to $U$

$$
\begin{aligned}
& U \vdash \mathbf{1} \stackrel{1}{\longrightarrow} \mathbf{0} \quad U \vdash \tau_{1} \rightarrow \tau_{2} \stackrel{\operatorname{ar}_{i}}{\stackrel{4}{*}} \tau_{i} \quad U \vdash \tau_{1} * \tau_{2} \stackrel{\operatorname{prd}_{i}}{\rightarrow} \tau_{i} \\
& \frac{U \vdash p \stackrel{\tau}{\rightarrow} p^{\prime}}{U \vdash p . t \stackrel{\tau}{\longrightarrow} p^{\prime} . t} \quad \frac{U \vdash p . t \mapsto\left(\theta, \text { sig } \ldots \text { type } t=\tau \ldots \text { end }^{i}\right)}{U \vdash p . t \stackrel{\tau}{\longrightarrow} \theta(\tau)} \\
& \frac{U \vdash p \mapsto\left(\theta, \text { sig } \ldots \text { datatype } t=c \text { of } \tau \ldots \text { end }^{i}\right)}{U \vdash p . t \stackrel{c}{-} \theta(\tau)} \\
& \frac{U \vdash X \mapsto\left(\theta, \text { sig ...type } t \ldots \text { end }^{i}\right)}{U \vdash X . t \stackrel{X . t}{\longrightarrow} \mathbf{0}}
\end{aligned}
$$

Figure 47: Transition rules for types with respect to $U$ 
Definitions and Specifications

$$
\begin{aligned}
& U \vdash_{X} E: T \\
& \frac{U \vdash_{X} E: T}{U \vdash_{X} \text { module } M=E: \text { module } M: T} \quad \frac{U \vdash_{X} S: T}{U \vdash_{X} \text { module } M: S: \text { module } M: T} \\
& \frac{U, \emptyset \vdash_{X} e: \tau}{U \vdash_{X} \operatorname{val} l=e: \operatorname{val} l: \tau} \quad \frac{U \vdash_{X} \tau \diamond U \vdash \tau \approx \tau^{\prime}}{U \vdash_{X} \operatorname{val} l: \tau: \operatorname{val} l: \tau^{\prime}} \\
& \overline{U \vdash_{X} \text { type } t: \text { type } t} \quad \overline{U \vdash_{X} \text { type } t: \text { type } t=\tau} \\
& \begin{array}{cc}
U \vdash_{X} \tau \diamond U \vdash \tau \approx \tau^{\prime} & U \vdash_{X} \tau \diamond U \vdash \tau \approx \tau^{\prime} \\
\hline \vdash_{X} \text { type } t=\tau: \text { type } t=\tau^{\prime}
\end{array} \\
& \frac{U \vdash_{X} E_{d}: T_{d}}{U \vdash_{X} E_{d}^{i}: T_{d}^{i}} \\
& \frac{U \vdash_{X} D_{1}: C_{1} \ldots U \vdash_{X} D_{n}: C_{n}}{U \vdash_{X} \text { struct }(Z) D_{1} \ldots D_{n} \text { end }: \operatorname{sig}(Z) C_{1} \ldots C_{n} \text { end }} \\
& U \vdash_{X} A: A^{\prime} \quad U \vdash_{X} E: T \\
& U \vdash_{X} \text { functor }(X: A) \rightarrow E: \text { functor }\left(X: A^{\prime}\right) \rightarrow T \\
& \frac{U \vdash_{X} E: T_{1} U \vdash_{X} S: T_{2} \quad U \vdash_{X} T_{1}<S}{U \vdash_{X}(E: S):\left(T_{1}: T_{2}\right)} \quad \frac{U \vdash_{X} p \text { wf }}{U \vdash_{X} p: p}
\end{aligned}
$$

Signature

$$
\frac{U \vdash_{X} S_{d}: T_{d}}{U \vdash_{X} S_{d}^{i}: T_{d}^{i}}
$$

Signature descriptions

$$
\begin{gathered}
\frac{U \vdash_{X} B_{1}: C_{1} \ldots U \vdash_{X} B_{n}: C_{n}}{U \vdash_{X} \operatorname{sig}(Z) B_{1} \ldots B_{n} \text { end }: \operatorname{sig}(Z) C_{1} \ldots C_{n} \text { end }} \\
\frac{U \vdash_{X} A: A^{\prime} \quad U \vdash_{X} S: T}{U \vdash_{X} \text { functor }(X: A) \rightarrow S: \text { functor }\left(X: A^{\prime}\right) \rightarrow T}
\end{gathered}
$$

Module variable signature

$$
\frac{U \vdash_{X} A_{d 1}: A_{d 2}}{U \vdash_{X} A_{d 1}^{i_{1}}: A_{d 2}^{i_{2}}}
$$

Module variable signature descriptions

$$
\frac{U \vdash_{X} B_{1}: C_{1} \ldots U \vdash_{X} B_{n}: C_{n}}{U \vdash_{X} \operatorname{sig} B_{1} \ldots B_{n} \text { end }: \operatorname{sig} C_{1} \ldots C_{n} \text { end }}
$$

Figure 48: Typing rules for the module language with respect to $U$ in TraviataX 


$$
\begin{aligned}
& \text { Core types } \\
& \overline{U \vdash_{X} \mathbf{1} \diamond} \frac{U \vdash_{X} \tau_{1} \diamond U \vdash_{X} \tau_{2} \diamond}{U \vdash_{X} \tau_{1} \rightarrow \tau_{2} \diamond} \quad \frac{U \vdash_{X} \tau_{1} \diamond U \vdash_{X} \tau_{2} \diamond}{U \vdash_{X} \tau_{1} * \tau_{2} \diamond} \\
& U \vdash_{X} p \text { wf } U \vdash_{Y} p \leadsto p^{\prime} \quad U \vdash p^{\prime} \mapsto\left(\theta, T_{d}^{i}\right) \\
& T_{d} \text { is a lazy signature type containing a type specification for } t \\
& U \vdash_{X} p . t \diamond \\
& \frac{U, \Gamma \vdash_{X} e: \tau^{\prime} \quad U \vdash \tau \approx \tau^{\prime}}{U, \Gamma \vdash_{X} e: \tau} \\
& \overline{U, \Gamma \vdash_{X}(): \mathbf{1}} \quad \frac{x \in \operatorname{dom}(\Gamma)}{U, \Gamma \vdash_{X} x: \Gamma(x)} \\
& \frac{U \vdash \tau \approx \tau_{1} \rightarrow \tau_{2} U \vdash_{X} \tau \diamond U, \Gamma, x: \tau_{1} \vdash_{X} e: \tau_{2}}{U, \Gamma \vdash_{X}(\lambda x . e: \tau): \tau} \quad \frac{U, \Gamma \vdash_{X} e_{1}: \tau_{1} \quad U, \Gamma \vdash_{X} e_{2}: \tau_{2}}{U, \Gamma \vdash_{X}\left(e_{1}, e_{2}\right): \tau_{1} * \tau_{2}} \\
& \frac{U, \Gamma \vdash_{X} e: \tau_{1} * \tau_{2}}{U, \Gamma \vdash_{X} \pi_{i}(e): \tau_{i}} \quad \frac{U, \Gamma \vdash_{X} e_{1}: \tau_{1} \rightarrow \tau_{2} \quad U, \Gamma \vdash_{X} e_{2}: \tau_{1}}{U, \Gamma \vdash_{X} e_{1}\left(e_{2}\right): \tau_{2}} \\
& \frac{U \vdash_{X} p \text { wf } U \vdash_{Y} p \leadsto p^{\prime} \quad \gamma_{X}\left(p^{\prime}, c, t\right)=\left(\tau_{1}, \quad\right) U, \Gamma \vdash_{X} e: \tau_{1}}{U, \Gamma \vdash_{X} p . c e: p^{\prime} . t} \\
& U \vdash_{X} p \text { wf } \quad U \vdash_{Y} p \leadsto p^{\prime} \\
& \frac{\gamma_{X}\left(p^{\prime}, c, t\right)=\left(\tau_{1},\right) U, \Gamma \vdash_{X} e_{1}: p^{\prime} . t \quad U, \Gamma, x: \tau_{1} \vdash_{X} e_{2}: \tau}{U, \Gamma \vdash_{X} \text { case } e_{1} \text { of } p . c x \Rightarrow e_{2}: \tau} \\
& \frac{U \vdash_{X} p \text { wf } U \vdash_{Y} p \leadsto p^{\prime} \quad U \vdash p^{\prime} \mapsto\left(\theta, \text { sig } \ldots \text { val }: \tau \ldots \text { end }^{i}\right)}{U, \Gamma \vdash_{X} p . l: \theta(\tau)} \\
& \frac{U \vdash_{X} \tau_{1} \diamond U \vdash_{X} \tau_{2} \diamond U, \Gamma \vdash_{X} e: \tau_{1} \quad U \vdash \tau_{1} \approx \tau_{2}}{U, \Gamma \vdash_{X}\left(e: \tau_{1}:: \tau_{2}\right): \tau_{2}}
\end{aligned}
$$

Figure 49: Typing rules for the core language with respect to $U$ in TraviataX

$$
\begin{aligned}
& \gamma_{X}(U, p, c)=(t, \theta(\tau)) \text { when } \\
& U \vdash p
\end{aligned}
$$

Figure 50: Datatype look-up with respect to $U$ in TraviataX 


$$
\begin{gathered}
\frac{}{U \vdash_{X} X \text { wf }} \quad \frac{U \vdash_{X} Z^{i d} \mathrm{wf}}{\frac{U \vdash_{Y} p . M \leadsto q U \vdash_{X} p \mathrm{wf}}{U \vdash_{X} p \cdot M \text { wf }}} \\
U \vdash_{X} p_{1} \text { wf } U \vdash_{X} p_{2} \mathrm{wf} \quad U \vdash_{Y} p_{1} \leadsto p_{1}^{\prime} U \vdash_{Y} p_{2} \leadsto p_{2}^{\prime} \\
\frac{U \vdash_{Y} p_{1}\left(p_{2}\right) \leadsto q \quad U \vdash p_{1}^{\prime} \mapsto\left(\theta,\left(\text { functor }\left(X: A_{d}^{j}\right) \rightarrow T\right)^{i}\right) \quad U \vdash_{X} p_{2}^{\prime}<\theta\left[X \mapsto p_{2}^{\prime}\right]\left(A_{d}\right)}{U \vdash_{X} p_{1}\left(p_{2}\right) \mathrm{wf}}
\end{gathered}
$$

Figure 51: Well-formed paths with respect to $U$ in TraviataX

$$
\begin{gathered}
\frac{U \vdash_{X} T_{d}<S_{d}}{U \vdash_{X} T_{d}^{i}<S_{d}^{j}} \quad \frac{U \vdash_{X} T_{d}<A_{d}}{U \vdash_{X} T_{d}^{i}<A_{d}^{j}} \\
\frac{U \vdash_{X} T_{d}<S_{d}}{U \vdash_{X}\left(T: T_{d}^{i}\right)<S_{d}} \\
\frac{U \vdash_{Y} p \leadsto p^{\prime} \quad U p^{\prime} \mapsto\left(\theta, T_{d}^{i}\right) \quad U \vdash_{X} \theta\left(T_{d}\right)<S_{d}}{U \vdash_{X} p<S_{d}} \\
\frac{\sigma:\{1, \ldots, m\} \mapsto\{1, \ldots, n\} \quad \forall i \in\{1, \ldots, m\}, U \vdash_{X} C_{\sigma(i)}<B_{i}}{U \vdash_{X} \operatorname{sig}(Z) C_{1} \ldots C_{n} \text { end }<\operatorname{sig}\left(Z^{\prime}\right) B_{1} \ldots B_{m} \text { end }} \\
\frac{U \vdash_{X} A^{\prime}<A \quad U \vdash_{X}\left[X \mapsto X^{\prime}\right] T<S}{U \vdash_{X} \text { functor }(X: A) \rightarrow T<\text { functor }\left(X^{\prime}: A^{\prime}\right) \rightarrow S} \\
\frac{\sigma:\{1, \ldots, m\} \mapsto\{1, \ldots, n\} \quad \forall i \in\{1, \ldots, m\}, U \vdash_{X} C_{\sigma(i)}<B_{i}}{U \vdash_{X} \operatorname{sig} C_{1} \ldots C_{n} \text { end }<\operatorname{sig} B_{1} \ldots B_{m} \text { end }}
\end{gathered}
$$

$\overline{U \vdash_{X} \text { type } t<\text { type } t} \quad \overline{U \vdash_{X} \text { type } t=\tau<\text { type } t} \quad \overline{U \vdash_{X} \text { datatype } t=c \text { of } \tau<\text { type } t}$

$$
\begin{aligned}
& \frac{U \vdash \tau_{1} \approx \tau_{2}}{U \vdash_{X} \text { type } t=\tau_{1}<\text { type } t=\tau_{2}} \quad \frac{U \vdash \tau_{1} \approx \tau_{2}}{U \vdash_{X} \operatorname{val} l: \tau_{1}<\operatorname{val} l: \tau_{2}} \\
& \frac{U \vdash \tau_{1} \approx \tau_{2}}{U \vdash_{X} \text { datatype } t=c \text { of } \tau_{1}<\text { datatype } t=c \text { of } \tau_{2}} \\
& \frac{U \vdash_{X} T<S}{U \vdash_{X} \text { module } M: T<\text { module } M: S}
\end{aligned}
$$

Figure 52: Subtyping with respect to $U$ in TraviataX 


\section{Type inference for the core language}

We implemented a type inference algorithm for the core language by determining an inference order using the module path expansion algorithm, then running a standard inference algorithm along this order. Concretely, using PathExp, we build a call graph of functions (represented by a directed graph), which expresses how functions in modules depend on each other: the strongly connected components of the graph indicate sets of value components whose type should be inferred simultaneously, referring to each other monomorphically; by topologically sorting the connected components, we generalize types in a connected component before moving on to typing the next one. For instance in Figure 2, we build an inference order:

Tree.split $\rightarrow$ \{Tree.labels, Forest.labels\}

$\rightarrow$ Forest.sweep $\} \rightarrow$ Forest.incr

where braces indicate strongly connected component. The inference order we build for Figure 1 is

$\{$ Tree.map $\rightarrow$ \{Forest.map

For the purpose of type inference, we do not consider that Tree.map and Forest.map are mutually recursive, since the signatures of Tree and Forest specify exported types for these functions.

We must also check for well-formedness of types, as module variables should not escape their scope during unification. This is checked after the inference. Note that when an abstract type depends on a functor argument, then the argument explicitly appears inside the type. For instance, in Figure 2, the type Tree.t is internally represented as $\mathrm{TF}^{[\mathrm{X} \mapsto \mathrm{x}]}$. Tree.t.

Explicit type annotations can be used to break dependencies in the call graph, and allow polymorphic recursion. Annotations cannot be completely avoided, as type inference for polymorphic recursion is known to be undecidable. 


\section{Related work}

Much work has been devoted to investigating recursive module extensions of the ML module system. Notably, type systems and initialization of recursive modules pose non-trivial issues, and have been the main subjects of study.

\subsection{Type systems}

To the best of our knowledge, no work has proposed a type system for recursive modules with applicative functors, except for the experimental implementation in Objective Caml [16], or examined type inference for recursive modules whether functors are applicative or generative. Moreover, only Traviata can type the examples on the expression problem in Section 6 without modifications.

The experimental implementation of recursive modules in Objective Caml is most related to our work. Indeed, we followed it in large part when designing Traviata. O'Caml supports a highly expressive core language and a strong type inference algorithm, which are one of our motivations for the effort to enable type inference. O'Caml also supports recursive signatures, with a rather concise syntax. However, it allows to write problematic modules whose type checking diverges. The potential for divergence when typing O'Caml modules is well-known, but is assumed to be a rare phenomenon in practice. Recursive signatures seem to make the problem much more acute. This is one of our motivations in insisting on decidable type checking for Traviata. Of course we obtain it through restrictions, and a less expressive signature language. Yet, this may be the price for safety. Since we have similar typing rules, we hope that our approach can apply to O'Caml with little change.

Crary, Harper and Puri [3] gave a foundational type theoretic analysis of recursive modules in the context of a phase-distinction formalism [11].

Russo $[24,23]$ proposed a type system for recursive modules, which we examined in Section 2.

Dreyer [5] gave a theoretical account for type abstraction inside recursive modules. In particular, he investigated generative functors in the context of recursive modules, by proposing a "destination passing" interpretation of type generativity. There is a critical difference in design choices between us, with respects to type abstraction inside recursive modules. For instance, consider the two programs:

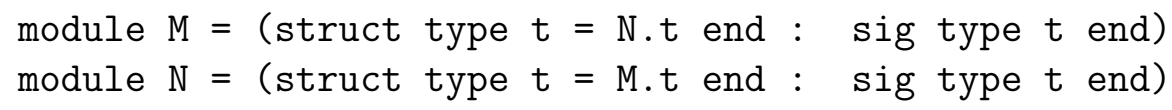


module $M=$ (struct type $t=N . t$ list end : sig type $t$ end)

module $N=$ (struct type $t=M . t * M . t$ end : sig type $t$ end)

Dreyer prohibits both programs, whereas we accept both. A motivation of our design choice is that we want to keep liberal uses of polymorphic variants and objects, which are useful constructs supported in O'Caml; prohibiting the latter program may result in restriction in using these constructs and recursive modules together.

\subsection{Initialization}

Boudol [1], Hirschowitz and Leroy [12], and Dreyer [4] have proposed type systems which ensure that initialization of recursive modules does not try to access components of modules that are not yet evaluated. They are interested in the safety of initialization, hence their modules do not have type components.

Their type systems judge the two modules:

module $M=\operatorname{struct~}(\mathrm{Z})$ val $\mathrm{l}=\mathrm{Z} \cdot \mathrm{m}$ val $\mathrm{m}=\mathrm{Z} . \mathrm{l}$ end

and

module $\mathrm{N}=\operatorname{struct}(\mathrm{Z})$ val $\mathrm{l}=\lambda \mathrm{x} \rightarrow \mathrm{x}+\mathrm{Z} \cdot \mathrm{m}$ val $\mathrm{m}=\mathrm{Z} . \mathrm{l}(3)$ end

to be ill-typed. In both cases, evaluation of the component $m$ cyclically requires evaluation of itself. Our type system, in particular the core type reconstruction algorithm, can reject the cycle for the former, but not for the latter. 


\section{Conclusion}

In this paper, we presented a type system for recursive modules by extending Leroy's applicative functor calculus. The type system is decidable and sound for a callby-value operational semantics. It supports type inference for recursive modules, hence type abstraction both inside and outside the recursion is handled equally; the programmer does not need to write two different signatures for the same module to assist the type checker.

We examined three examples. The first two presented typical uses of recursive modules with different choices of where to enforce type abstraction. The last one gave a solution to the expression problem and demonstrated how recursive modules add to the expressive power of the programming language when combined with other language constructions.

Here we give a brief overview of future work.

Separate type checking Although we have not discussed, Traviata is already prepared for separate type checking. In short, we only have to extend the look-up judgment (Figure 7) so that the judgment informs the type system of signatures of modules which are type checked separately(i.e., to replace concrete module expressions with their signatures).

Lazy modules with eager value components The operational semantics presented in this paper uses lazy evaluation for both modules and their value components in the sense that only components of modules that are accessed are evaluated, and the evaluation is triggered at access time. This semantics simplifies the soundness statement and its proof. For a practical system, however, we are investigating lazy modules with eager value components, that is, to keep modules lazy but evaluate all the value components (but not module components) of a module at once, triggered by the first access to some component of the module. Lazy semantics of modules would allow flexible uses of recursive modules; eager semantics of value components would give the programmer a way to initialize recursive modules.

We need to explore the evaluation strategy of modules. For instance, we want to succeed in evaluating the following modules whether the evaluation begins at $\mathrm{M}$ or at N.

module $\mathrm{M}=$ struct val $\mathrm{m} 1=3$ val $\mathrm{m} 2=\mathrm{N} . \mathrm{n} 1$ val $\mathrm{m} 3=7$ end

module $\mathrm{N}=$ struct val $\mathrm{n} 1=5$ val $\mathrm{n} 2=\mathrm{M} . \mathrm{m} 1$ val $\mathrm{n} 3=\mathrm{M} . \mathrm{m} 3$ end

We also need to cope with unsafe modules whose evaluation cannot be successful, 
for instance the modules we saw in Section 10.2; we want to avoid running into divergence but to reject them statically or, at least, to raise run-time errors.

We believe that our algorithms for path resolution are useful for efficient and safe implementation of lazy recursive modules. We need more investigation on this topic.

The double vision problem It is desirable to solve the double vision problem without requiring type coercion annotations from the programmer. The current type system always passes to TypExp the whole lazy program type ReconstP constructed. This seems too naïve. Given that TypExp terminates for whatever input, we think it is safe to pass TypExp different signature information depending on whether it is used inside sealing or not. For instance in Figure 26, we should make TypExp interpret the sealing signature of Forest transparently during type checking inside Forest.

\section{References}

[1] G. Boudol. The recursive record semantics of objects revisited. Journal of Functional Programming, 14(3):263-315, 2004.

[2] W. R. Cook. Object-Oriented Programming Versus Abstract Data Types. In Proc. REX Workshop, volume 489 of Lecture Notes in Computer Science. Springer-Verlag, 1990.

[3] K. Crary, R. Harper, and S. Puri. What is a Recursive Module? In ACM SIGPLAN Conference on Programming Language Design and Implementation. ACM Press, 1999.

[4] D. Dreyer. A type system for well-founded recursion. In ACM SIGPLAN Symposium on Principles of Programming Languages. ACM Press, 2004.

[5] D. Dreyer. Recursive Type Generativity. In ACM SIGPLAN International Conference on Functional Programming. ACM Press, 2005.

[6] D. Dreyer. Understanding and Evolving the ML Module System. PhD thesis, Carnegie Mellon University, 2005.

[7] J. Garrigue. Programming with polymorphic variants. In ACM SIGPLAN Workshop on $M L, 1998$.

[8] J. Garrigue. Code reuse through polymorphic variants. In Workshop on Foundations of Software Engineering, 2000. 
[9] J. Garrigue. Private rows: abstracting the unnamed. http://www.math. nagoya-u.ac.jp/ garrigue/papers/privaterows.pdf, 2005.

[10] R. Harper and M. Lillibridge. A Type-Theoretic Approach to Higher-Order Modules with Sharing. In ACM SIGPLAN Symposium on Principles of Programming Languages, pages 123-137, 1994.

[11] R. Harper, J. C. Mitchell, and E. Moggi. Higher-order modules and the phase distinction. In ACM SIGPLAN Symposium on Principles of Programming Languages, pages 341-354, 1990.

[12] T. Hirschowitz and X. Leroy. Mixin modules in a call-by-value setting. In European Symposium on Programming:LNCS, volume 2305, pages 6-20. SpringerVerlag, 2002.

[13] X. Leroy. Manifest types, modules, and separate compilation. In ACM SIGPLAN Symposium on Principles of Programming Languages. ACM Press, 1994.

[14] X. Leroy. Applicative functors and fully transparent higher-order modules. In ACM SIGPLAN Symposium on Principles of Programming Languages. ACM Press, 1995.

[15] X. Leroy. A modular module system. Journal of Functional Programming, 10(3):269-303, 2000.

[16] X. Leroy, D. Doligez, J. Garrigue, and J. Vouillon. The Objective Caml system, release 3.09. Software and documentation available on the Web, http://caml. inria.fr/, 2005.

[17] D. MacQueen. Modules for Standard ML. In Proc. the 1984 ACM Conference on LISP and Functional Programming, pages 198-207. ACM Press, 1984.

[18] R. Milner. communicating and mobile systems: the pi-calculus. Cambridge University Press, 1999.

[19] R. Milner, M. Tofte, R. Harper, and D. MacQueen. The Definition of Standard ML (Revised). MIT Press, 1997.

[20] K. Nakata and J. Garrigue. Path resolution for recursive modules. Technical Report 1545, Kyoto University Research Institute for Mathematical Sciences, 2006. 
[21] B. Pierce, editor. Advanced Topics in Types and Programming Languages, chapter 9. The MIT Press, 2004.

[22] D. Rémy and J. Garrigue. On the expression problem. http://pauillac. inria.fr/ ${ }^{\sim}$ remy/work/expr/, 2004.

[23] S. Romanenko, C. Russo, N. Kokholm, and P. Sestoft. Moscow ML, 2004. Software and documentation available on the Web, http://www.dina.dk/ sestoft/mosml.html.

[24] C. Russo. Recursive Structures for Standard ML. In ACM SIGPLAN International Conference on Functional Programming. ACM Press, 2001.

[25] D. Sangiorgi and D. Walker. The Pi-Calculus. Cambridge University Press, 2003.

[26] M. Torgersen. The Expression Problem Revisited. In European Conference on Object-Oriented Programming:LN CS, volume 3086. Springer-Verlag, 2004.

[27] P. Wadler. The expression problem. Java Genericity maling list, 1998. http: //www.cse.ohio-state.edu/ gb/cis888.07g/java-genericity/20.

[28] M. Zenger and M. Odersky. Independently Extensible Solutions to the Expression Problem. In Proc. FOOL 12, 2005. 